๑ IEEE $\mid \begin{aligned} & \text { Transactions on } \\ & \text { Wireless Communications }\end{aligned}$

\title{
Coverage Analysis of Multi-Stream MIMO HetNets with MRC Receivers
}

\begin{tabular}{|r|l|}
\hline Journal: & IEEE Transactions on Wireless Communications \\
\hline Manuscript ID & Paper-TW-Mar-17-0285 \\
\hline Manuscript Type: & Original Transactions Paper \\
\hline Complete List of Authors: & $\begin{array}{l}\text { Khoshkholgh, Mohammad; The University of British Columbia, EECE; } \\
\text { Navaie, Keivan; Lancaster University, School of Computing and } \\
\text { Communications } \\
\text { Shin, Kang G. ; University of Michigan, EECS } \\
\text { Leung, Victor; The University of British Columbia, Electrical and Computer } \\
\text { Engineering }\end{array}$ \\
\hline Keyword: &
\end{tabular}




\title{
Coverage Analysis of Multi-Stream MIMO HetNets
}

\section{with MRC Receivers}

\author{
Mohammad G. Khoshkholgh, Keivan Navaie, Senior Member, IEEE, \\ Kang. G. Shin, Life Fellow, IEEE, Victor C. M. Leung, Fellow, IEEE
}

\begin{abstract}
Most of current research on the coverage performance of multi-stream MIMO heterogeneous networks (HetNets) has been focusing on a single data-stream. This does not always provide accurate results as our analysis shows the cross-stream correlation due to interference can greatly affect the coverage performance. This paper analyzes the coverage probability in such systems, and studies the impact of cross-stream correlation. Specifically, we focus on the max-SIR cell association policy, and leverage stochastic geometry to study scenarios whereby a receiver is considered in the coverage, if all of its data-streams are successfully decodeable. Assuming open-loop maximum ratio combining (MRC) at receivers, we consider cases where partial channel state information is available at the receiver. We then obtain an upper-bound on the coverage and formulate cross-stream SIR correlation. We further show that approximating such systems based on fully-correlated (non-correlated) data-streams, results in a slight underestimation (substantial overestimation) of the coverage performance. Our results provide insights on the multiplexing regimes where densification improves the coverage performance. We also compare MRC with more complex zero-forcing receiver and provide quantitative insights on the design trade-offs. Analysis is validated via extensive simulations.
\end{abstract}

\section{Index Terms}

Manuscript received March 06, 2017. Part of this work has been presented in the IEEE VTC-F 2016, Montreal [1]. M. G. Khoshkholgh and V. C. M. Leung (m.g.khoshkholgh@gmail.com,vleung@ece.ubc.ca) are with the Department of Electrical and Computer Engineering, the University of British Columbia, Vancouver, BC, Canada V6T 1Z4; K. Navaie (k.navaie@lancaster.ac.uk) is with the School of Computing and Communications, Lancaster University, Lancaster, UK LA1 4WA; K. G. Shin (kgshin@umich.edu) is with the Department of Electrical Engineering and Computer Science, University of Michigan, Ann Arbor, MI 48109-2121, U.S.A. 


\begin{abstract}
Densification, heterogeneous networks (HetNets), multiple-input multiple-output (MIMO), multiplexing gain, network-wise coverage performance, signal-to-interference ratio (SIR) correlation, stochastic geometry.
\end{abstract}

\title{
I. INTRODUCTION
}

Spectral efficiency in heterogenous networks (HetNets) is substantially enhanced using densification and universal frequency reuse. A key physical-layer component of dense HetNets is MIMO technology which is also capable of meeting the high demand for wireless bandwidth $[2,3]$. Nevertheless, macroscopic (network-level) performance, where MIMO multiplexing communication is utilized in conjunction with densification and heterogeneity, still remains to be explored.

Conventionally, MIMO systems are analyzed for isolated scenarios, where only point-to-point, single cell, and/or clustered communications are considered $[4,5]$. Such analyses can characterize the various design aspects of MIMO HetNets, but they cannot capture the macroscopic performance of MIMO systems under severe and heterogenous inter-cell interference (ICI), commonly seen in dense HetNets with aggressive frequency reuse. We would like to address this very issue by using stochastic-geometry-based analytical techniques.

Stochastic geometry has been widely used for modeling and performance evaluation of wireless cellular networks, including HetNets, e.g., [6-10]. Using these techniques enables incorporation of impacts of line-of-sight propagation, path-loss models, and blockage effect into the networkwise evaluation of spectral efficiency without compromising the tractability and accuracy of the analysis $[8,10,11]$.

\section{A. Related Work}

Reviewed below are the related studies of the performance of MIMO systems. The adopted cell-association (CA) policy plays a crucial role in the performance of MIMO HetNet systems. In a given coverage area, cell association determines which BS to serve a given mobile user. Different CA approaches are categorized as range expansion and Max-SIR association.

The range expansion policy uses maximum average received power as the association criterion. The coverage probability and area spectral efficiency (ASE) of multiple-input single-output 
(MISO) space-division multiple access (SDMA) systems utilizing this CA policy has been investigated in $[12,13]$. The merits of interference cancellation in zero-forcing (ZF) based receive filters in enhancing the coverage of cellular systems was also demonstrated in [14]. Further, in [15-20] design issues, impacts of beamforming schemes, and antenna selection techniques on ASE, coverage, and energy-efficiency of MIMO communications with range extension CA were investigated [15-17]. Optimized offloading for controlling ICI utilizing coordinated MIMO communications was carried out in [18]. Coverage probability, spectral efficiency, and load balancing in MIMO systems are also considered in [19]. To improve the performance of range expansion in MIMO systems, the authors of [20] proposed a threshold-based CA solution.

With multi-antenna receivers, the authors of [21,22] focused on maximum ratio combining (MRC) and optimal combining in the downlink and uplink, respectively. Applying Gil-Pelaez inversion theorem in [11], the symbol error probability (SEP) of MIMO multiplexing systems was analyzed in [23]. Equivalent-in distribution (EiD) was also developed in [24] to quantify error probability. Adopting the proposed framework of [24], a unified method for studying outage probability in MIMO communications was then proposed in [25].

In general, range-expansion CA does not distinguish between the corresponding MIMO techniques in the CA stage. So, in many cases mentioned above, the CA policy is in fact a replica of the one considered in the SISO counterpart [7,9]. This makes range expansion defiant in effectively incorporating the attributes of MIMO communications to improve multiplexing and diversity. That is one of the reasons why heuristic offloading procedures are often required to optimize the system performance $[13,18,20]$.

Nevertheless, the coverage is directly related to signal-to-interference (SIR) distribution. In addition, many network management functions, such as handover and fractional frequency reuse, often operate based on the SIR (or a function thereof) as the main decision metric. These justify consideration of CA rules based on the SIR characteristics.

The authors of $[6,26,27]$ considered max-SIR CA in which the serving BS is the one that provides the maximum SIR. For MISO systems, the authors of [28] provided ordering results on the coverage, capacity, and ASE, and compared several beamforming techniques. In [29], 
we proposed a flexible max-SIR CA rule tailored for MISO-SDMA systems. Algorithms for specifying the number of required SIR measurements before choosing the supporting BS in order to optimize the coverage probability/spectral efficiency were also developed in [29].

Unlike the range-expansion technique for which various aspects of coverage performance have been investigated, the coverage performance of multi-stream MIMO-MRC communications with max-SIR CA is yet to be explored. The main objective of this paper is to analyze the coverage performance of MIMO communication with max-SIR CA rule, where multiple streams are transmitted at the same time. Note that in the literature of multi-stream MIMO communications, the coverage probability of the network is often estimated from the perspective of a given data stream. The thus-obtained coverage for a given data stream is then treated as the coverage performance of the multiplexing (multi-stream) system, see, e.g., [21,30-33]. Nevertheless, such approach may cause substantial error in the evaluation of the coverage probability of multi-stream MIMO HetNets, as the possible correlation across data streams are entirely overlooked.

In fact, when the SIR values among data streams are correlated, the stream-level performance that considers the reception quality of a single data stream independent from the others, becomes inadequate. This is because the successful decoding of a data stream is partially dependent upon the decoding status of other data streams. Therefore, the coverage performance of MIMO multiplexing systems from a link-level perspective that considers the reception of all the data steams becomes crucial. In our previous works, [1][49][34], we studied the coverage probability of MIMO multiplexing systems from a link-level perspective. In [49] the focus was on multistream MIMO systems where the pre-coding and decoding filters at the transmitter and receiver was constructed according the singular value decomposition (SVD) technique. This techniques however requires perfect and timely CSI at both the transmitter and receiver, which imposes high signaling overhead particularly in dense configurations. Furthermore, in [34], we investigated the link-level coverage performance for multi-stream MIMO networks with zero-forcing beamforming (ZFBF) receivers. The simulation results in [34] show subtle differences between link-level, and stream-level coverage performance in a multi-stream MIMO system. Despite its importance, to the best of our knowledge, the roots and scales of such a discrepancy has not yet 
been investigated in the related literature.

On the other hand, in SIMO ad hoc networks, the ICI is shown to result in a high correlation among impinged signals across different receive antennas, see, e.g., [35, 36]. Such a correlation compromise the otherwise achievable diversity gain in cases where signals across antennas are independent. This is because in the presence of ICI, the path-loss fluctuations invoke (statistically) correlated interference among antennas due to the common locations of interferers. A similar conclusion was drawn in [21], where the interference correlation was investigated in spacetime MIMO ad hoc networks. It was also shown in [21] that ignoring interference correlation among antennas may, in some cases, substantially compromise the accuracy of the analysis. The analysis in [21] is, however, limited to the CDF distribution of an individual data stream, thus being unable to depict the impact of correlation on the CDF distribution of a communication link with a set of data streams.

\section{B. Contributions and Organization}

In this paper, we investigate cross-stream SIR correlation and its impacts on the link-level coverage probability in MIMO multiplexing systems. We mainly focus on the maximum ratio combining (MRC) receivers. Note that compared to the ZFBF, the coverage evaluation of the MRC is more challenging due to the cross-stream interference. The coverage performance of MIMO-MRC systems from the stream-level perspective is studied in the context of ad hoc communications, e.g., [32]. The results in an ad hoc context are not necessarily extendable to cellular networks because, unlike cellular systems, ad hoc communications often operate without a CA mechanism and lack a central scheduler.

Here we evaluate the MIMO-MRC coverage probability from a link-level perspective in cellular networks. Despite the popularity and practical significance of an MIMO-MRC system for cellular communications due to its simple implementation and near zero feedback overheads, its performance in HetNet settings has not yet been investigated. Our model and analysis are concerned with scenarios that channel state information (CSI) is not available at the BSs and only partially known at the UEs. This paper makes the following two main contributions. 
- We obtain a closed-form and easy-to-compute tight upper bound on the network coverage probability for cases where successful decoding of all transmitted data streams is required. The unique feature of our analysis is to accurately incorporate SIR correlation. Our analytical results_-supported by extensive simulations-provide significant practical insights on the impacts of densification on the link-level coverage performance. Based on this result, we conclude that improvement in the network coverage performance by densification is subject to careful selection of multiplexing gains in different tiers.

- We also analyze the cross-stream SIR correlation amongst multiple streams in a communication link. Our analysis provides quantitative insights on the impact of tiers' BSs density, pathloss exponent, CSI inaccuracy, and multiplexing gains on the SIR correlation among data streams. To understand the impacts of SIR correlation on the coverage probability, we then obtain the closed-form bounds on the coverage probabilities for two extreme settings: full SIR correlation (FC) among data streams, and no SIR correlation (NC) among data streams. We then show that the NC setting substantially over-estimates the coverage performance while the FC setting slightly underestimates it.

The rest of the paper is organized as follows. Section II presents the system model and Section III provides coverage evaluation. Section IV investigates the SIR correlation and its impact on the coverage probability. The simulation results are provided in Section $\mathrm{V}$ followed by conclusions in Section VI.

\section{SySTEM MODEL}

We consider the downlink in a heterogeneous cellular network (HetNet) consisting of $K \geq 1$ tiers of randomly located base-stations (BSs). In each tier $i \in \mathcal{K}$, BSs are spatially distributed according to a homogenous Poisson Point Process (PPP), $\Phi_{i}$, with a given spatial density, $\lambda_{i} \geq$ 1 [6]. For mathematical tractability, we assume that the PPPs corresponding to each tier are mutually independent. Therefore, each tier $i$ can be completely characterized by the spatial density of its BSs, $\lambda_{i}$, their transmit power, $P_{i}$ Watts, the corresponding SIR threshold at the receivers, $\beta_{i} \geq 1$, the number of BS's transmit antennas $N_{i}^{t}$, and the number of scheduled streams $S_{i} \leq \min \left\{N_{i}^{t}, N^{r}\right\}$ (also referred to as the multiplexing gain), where $N^{r}$ is the number 
of antennas at the user equipments (UEs).

In the model under consideration, $S_{i}$ data streams are considered in each tier/BS as parallel flows of information as in [31,32]. UEs are randomly located across the network coverage area and form a PPP, $\Phi_{U}$, with density $\lambda_{U} \gg \sum_{i} \lambda_{i}$, independent of $\left\{\Phi_{i}\right\}$ s. Similar to $[21,28,37]$, we further assume that in each active cell, only one UE is served at each time slot. If more than one UE are associated with a given BS, we adopt time-sharing per cell for scheduling the UEs. Considering the stationarity of the point processes, according to Slivnayak's theorem, we can investigate spatial network performance from the perspective of a UE located at the origin [38, 39]; we will refer to such an UE the typical UE.

Let $\boldsymbol{H}_{x_{i}} \in \mathbb{C}^{N^{r} \times S_{i}}$ be the fading channel matrix between BS $x_{i}$ and the typical UE, where each entry is independently drawn from a complex Gaussian random variable with zero mean and unit variance, $\mathcal{C N}(0,1)$, i.e., Rayleigh fading assumption.

Here we focus on the scenarios that only partial CSI is available at the receivers. As in $[40,41]$, the quantified measure for channel estimation error is considered to be the correlation coefficient between the actual fading channel coefficient and its estimated value as $\boldsymbol{H}_{x_{i}}=$ $\sqrt{1-\epsilon_{i}^{2}} \widetilde{\boldsymbol{H}}_{x_{i}}+\epsilon_{i} \boldsymbol{E}_{x_{i}}$, where $\widetilde{\boldsymbol{H}}_{x_{i}}$ is the estimated channel which is a complex Gaussian random matrix with zero mean and identity covariance matrix; $\epsilon_{i}^{2}$ measures the inaccuracy of channel estimation; and $\boldsymbol{E}_{x_{i}}$ is a complex Gaussian random matrix with zero mean and identity covariance matrix. Random variables $\boldsymbol{E}_{x_{i}}$ and $\widetilde{\boldsymbol{H}}_{x_{i}}$ are assumed independent, e.g., in cases where CSI is estimated using a pilot-based minimum mean square error (MMSE) [40,41].

For the typical UE associated with BS $x_{i}$ transmitting $S_{i}$ data streams, the received signal, $\boldsymbol{y}_{x_{i}} \in \mathbb{C}^{N^{r} \times 1}$, is:

$$
\boldsymbol{y}_{x_{i}}=\left\|x_{i}\right\|^{-\frac{\alpha}{2}} \sqrt{1-\epsilon_{i}^{2}} \widetilde{\boldsymbol{H}}_{x_{i}} \boldsymbol{s}_{x_{i}}+\left\|x_{i}\right\|^{-\frac{\alpha}{2}} \epsilon_{i} \boldsymbol{E}_{x_{i}} \boldsymbol{s}_{x_{i}}+\sum_{j \in \mathcal{K}} \sum_{x_{j} \in \Phi_{j} / x_{0}}\left\|x_{i}\right\|^{-\frac{\alpha}{2}} \boldsymbol{H}_{x_{j}} \boldsymbol{s}_{x_{j}}
$$

where $\boldsymbol{s}_{x_{i}}=\left[s_{x_{i}, 1} \ldots s_{x_{i}, S_{i}}\right]^{T} \in \mathbb{C}^{S_{i} \times 1}$, so that $s_{x_{i}, l} \sim \mathcal{C N}\left(0, P_{i} / S_{i}\right)$, is the transmitted streams at $\mathrm{BS} x_{i} ;\left\|x_{i}\right\|^{-\alpha}$ is the distance-dependent path-loss attenuation; $\left\|x_{i}\right\|$ denotes the Euclidian distance between BS $x_{i}$ and the origin; and $\alpha>2$ is the path-loss exponent. We further assume that the transmitted signals as well as channel matrices are independent. The first term in (1) accounts for the useful signal, the second term represents the interference due to inaccuracy of CSI, and the last term is the ICI. At the receiver, maximum ratio combining (MRC) [32] is 
adopted with decoding filter $\boldsymbol{U}_{x_{i}}=\boldsymbol{H}_{x_{i}}=\left[\boldsymbol{h}_{x_{i}, 1} \ldots \boldsymbol{h}_{x_{i}, S_{i}}\right]$. Post-processing SIR for data stream $l_{i}$ is therefore

$$
\operatorname{SIR}_{x_{i}, l_{i}}^{\mathrm{MRC}}=\frac{\frac{P_{i}}{S_{i}}\left\|x_{i}\right\|^{-\alpha}\left(1-\epsilon_{i}^{2}\right)\left\|\tilde{\boldsymbol{h}}_{x_{i}, l_{i}}\right\|^{2}}{\frac{P_{i}}{S_{i}\left\|x_{i}\right\|^{\alpha}}\left(\sum_{l \prime \neq l_{i}} \frac{\left\|\tilde{\boldsymbol{h}}_{x_{i}, l_{i}}^{\dagger} \boldsymbol{h}_{x_{i}, l^{\prime}}\right\|^{2}}{\left\|\tilde{\boldsymbol{h}}_{x_{i}, l_{i}}\right\|^{2}}+\epsilon_{i}^{2} \frac{\left\|\tilde{\boldsymbol{h}}_{x_{i}, l_{i}}^{\dagger} \boldsymbol{e}_{x_{i}, l_{i}}\right\|^{2}}{\left\|\tilde{\boldsymbol{h}}_{x_{i}, l_{i}}\right\|^{2}}\right)+\sum_{j \in \mathcal{K}} \sum_{x_{j} \in \Phi_{j} / x_{i}} \frac{P_{j}}{S_{j}\left\|x_{j}\right\|^{\alpha}} \sum_{l_{j}=1}^{S_{j}} \frac{\| \tilde{\boldsymbol{h}}_{x_{i}, l_{i}}^{\dagger} \boldsymbol{h}_{x_{j}, l_{j} \|^{2}}}{\| \tilde{\boldsymbol{h}}_{x_{i}, l_{i} \|^{2}}}} .
$$

We then set random variable (r.v.) $H_{x_{i}, l_{i}}^{\mathrm{MRC}} \triangleq\left\|\tilde{\boldsymbol{h}}_{x_{i}, l_{i}}\right\|^{2}$ which is chi-squared with $2 N^{r}$ degreesof-freedom (DoFs). Further, we define r.v.s $\hat{H}_{x_{i}, l_{i}}^{\mathrm{MRC}} \triangleq \sum_{l^{\prime} \neq l_{i}} \frac{\left\|\tilde{\boldsymbol{h}}_{x_{i}, l_{i}}^{\dagger} \boldsymbol{h}_{x_{i}, l^{\prime}}\right\|^{2}}{\left\|\tilde{\boldsymbol{h}}_{x_{i}, l_{i}}\right\|^{2}}$ which is also chi-squared with $2\left(S_{i}-1\right)$ DoFs, and $\tilde{H}_{x_{i}, l_{i}}^{\mathrm{MRC}} \triangleq \frac{\left\|\tilde{\boldsymbol{h}}_{i_{i}, l_{i}}^{\dagger} \boldsymbol{e}_{x_{i}, l_{i}}\right\|^{2}}{\left\|\tilde{\boldsymbol{h}}_{x_{i}, l_{i}}\right\|^{2}}$ which is an exponential r.v. Both $\hat{H}_{x_{i}, l_{i}}^{\mathrm{MRC}}$ and $\tilde{H}_{x_{i}, l_{i}}^{\mathrm{MRC}}$ are independent of $H_{x_{i}, l_{i}}^{\mathrm{MRC}}$. We further set $G_{x_{j}, l_{i}}^{\mathrm{MRC}} \triangleq \sum_{l_{j}=1}^{S_{j}} \frac{\left\|\tilde{\boldsymbol{h}}_{x_{i}, l_{i}}^{\dagger} \boldsymbol{h}_{x_{j}, l_{j}}\right\|^{2}}{\left\|\tilde{\boldsymbol{h}}_{x_{i}, l_{i}}\right\|^{2}}$ which is also chisquared with $2 S_{j}$ DoFs and independent of $\hat{H}_{x_{i}, l_{i}}^{\mathrm{MRC}}, \tilde{H}_{x_{i}, l_{i}}^{\mathrm{MRC}}$, and $H_{x_{i}, l_{i}}^{\mathrm{MRC}}$. Using the above notation, $H_{x_{i}, l_{i}}^{\mathrm{MRC}}, \hat{H}_{x_{i}, l_{i}}^{\mathrm{MRC}}, \tilde{H}_{x_{i}, l_{i}}^{\mathrm{MRC}}$, and $G_{x_{j}, l_{i}}^{\mathrm{MRC}}$, respectively, stand for the channel power gains associated with the intending the $l_{i}$-th data stream, the interference on stream $l_{i}$ due to imperfect CSI estimation, the inter-stream interference caused by streams $l_{i}^{\prime} \neq l_{i}$, and the ICI imposed by $x_{j} \neq x_{i}$. Postprocessing SIR in (2) is then represented as

$$
\operatorname{SIR}_{x_{i}, l_{i}}^{\mathrm{MRC}}=\frac{\frac{P_{i}}{S_{i}}\left\|x_{i}\right\|^{-\alpha}\left(1-\epsilon_{i}^{2}\right) H_{x_{i}, l_{i}}^{\mathrm{MRC}}}{\frac{P_{i}}{S_{i}\left\|x_{i}\right\|^{\alpha}}\left(\hat{H}_{x_{i}, l_{i}}^{\mathrm{MRC}}+\epsilon_{i}^{2} \tilde{H}_{x_{i}, l_{i}}^{\mathrm{MRC}}\right)+\sum_{j \in \mathcal{K}} \sum_{x_{j} \in \Phi_{j} / x_{i}} \frac{P_{j} G_{x_{j}, l_{i}}^{\mathrm{MRC}}\left\|x_{j}\right\|^{\alpha}}{S^{2}}} .
$$

Eq. (3) incorporates per-stream transmission power, multiplexing gains, ICI, CSI inaccuracy, and inter-stream interference.

\section{Coverage Performance Evaluation}

\section{A. Coverage Performance in Multi-Stream MIMO Systems}

In HetNets, similar to other wireless networks, the SIR is translated into practical performance metrics, such as the coverage probability. For a given coverage probability, one can then, among other parameters, evaluate the required density of the BSs in each tier and/or their multiplexing gains. In the case of a HetNet with single-stream transmission, the coverage probability in a tier, $i$, is directly related to the cumulative distribution function (CDF) of the corresponding SIR. More specifically, for tier $i$, the coverage probability is often defined as the probability that the SIR stays above a given threshold, $\beta_{i}$, throughout the coverage area. In the case of multiple streams however, depending on the transceiver structure and/or the quality requirements, evaluating the coverage probability becomes more complex. 
In some transceiver techniques, the coverage probability depends upon the CDF of the weakest SIR value among $S_{i}$ streams [42-45]. Thus, a UE is considered in the coverage if all of its streams are successfully decoded; this is referred to all-coverage probability as in the isolated scenarios $[45,46] .^{1}$

To specify the CA policy, we focus on the max-SIR CA rule as in $[6,28,34]$, where a typical UE is associated with a BS that provides the strongest SIR. To evaluate the all-coverage probability, we adopt the max-SIR CA rule of [34] which is an extension of the one considered in $[6,28,29]$, to the multi-stream MIMO communications: the associated BS is the one whose corresponding minimum SIR value (measured across streams) is the maximum among all the BSs. For brevity, we will henceforth refer to the all-coverage probability as the coverage performance. A typical UE is thus in the coverage if the set

$$
\mathcal{A}_{\mathrm{all}}^{\mathrm{MRC}}=\left\{\exists i \in \mathcal{K}: \max _{x_{i} \in \Phi_{i} l_{i}=1, \ldots, S_{i}} \min _{x_{i}, l_{i}}^{\mathrm{MRC}} \geq \beta_{i}\right\},
$$

is nonempty and the coverage probability is defined as $\mathcal{P}_{C}^{\mathrm{MRC}}=\mathbb{P}\left\{\mathcal{A}_{\text {all }}^{\mathrm{MRC}} \neq \emptyset\right\}$.

\section{B. The Coverage Probability}

Analytical evaluation of $\mathcal{P}_{C}^{\mathrm{MRC}}$ is rather complex due mainly to the cross stream SIR correlation, non-Rayleigh-type fluctuations, CSI inaccuracy, and also the inter-stream interference. In the following proposition, we provide an analytical upper bound on the coverage probability.

Proposition 1: In a MIMO-MRC system adopting maximum SIR CA rule, the coverage probability is upper-bounded as:

$$
\mathcal{P}_{C}^{\mathrm{MRC}} \leq \frac{\pi}{\tilde{C}(\alpha)} \sum_{i \in \mathcal{K}} \frac{\lambda_{i}\left(\frac{P_{i}\left(1-\epsilon_{i}^{2}\right)}{S_{i}^{2} \beta_{i}}\right)^{\check{\alpha}}\left(\Theta\left(\beta_{i}, \epsilon_{i}, S_{i}\right)\right)^{S_{i}}}{\sum_{j=1}^{K} \lambda_{j}\left(\frac{P_{j}}{S_{j}}\right)^{\check{\alpha}}\left(\frac{\Gamma\left(\frac{\check{\alpha}}{S_{i}}+S_{j}\right)}{\Gamma\left(S_{j}\right)}\right)^{S_{i}}},
$$

where

$$
\Theta\left(\beta_{i}, \epsilon_{i}, S_{i}\right) \triangleq \sum_{r_{i}=0}^{N^{r}-1} \sum_{q_{i}=0}^{r_{i}} \sum_{p_{i}=0}^{q_{i}} \frac{(-1)^{q_{i}-p_{i}} \beta_{i}^{2 q_{i}-p_{i}}}{\epsilon_{i}^{-4 q_{i}+2 p_{i}}\left(1-\epsilon_{i}^{2}\right)^{S_{i}}} \frac{\left(1-\epsilon_{i}^{2}+\beta_{i}\right)^{-q_{i}-S_{i}+1}\left(1+\epsilon_{i}^{2}\left(\beta_{i}-1\right)\right)^{-q_{i}+p_{i}-1}}{p_{i} \mathrm{~B}\left(S_{i}-1, p_{i}\right)\left(r_{i}-q_{i}\right) \mathrm{B}\left(\frac{\check{\alpha}}{S_{i}}, r_{i}-q_{i}\right)},
$$

and $\mathrm{B}(a, b)=\frac{\Gamma(a) \Gamma(b)}{\Gamma(a+b)}$ is the beta function.

\footnotetext{
${ }^{1}$ Note that if the original data streams are spatially coded across multiple data streams, then sum-coverage probability is a relevant metric, whereby the accumulated transmitted data rate must be large enough for a link to be considered in coverage $[45,47]$. We exclude such cases and focus on the all-coverage probability.
} 
Proof: We use Lemma 1 in [6] and note that $\beta_{i} \geq 1, \forall i$, and write

$$
\begin{gathered}
\mathcal{P}_{C}^{\mathrm{MRC}}=\sum_{i \in \mathcal{K}} \mathbb{E} \sum_{x_{i} \in \Phi_{i}} 1\left(\min _{l_{i}=1, \ldots, S_{i}} \operatorname{SIR}_{x_{i}, l_{i}}^{\mathrm{MRC}} \geq \beta_{i}\right)=\sum_{i \in \mathcal{K}} 2 \pi \lambda_{i} \int_{0}^{\infty} x_{i} \mathbb{E}_{\left\{\Phi_{j}\right\}} \mathbb{P}\left\{\operatorname{SIR}_{x_{i}, l_{i}}^{\mathrm{MRC}} \geq \beta_{i}: \forall l_{i} \mid\left\{\Phi_{j}\right\}\right\} d x_{i} \\
=\sum_{i \in \mathcal{K}} 2 \pi \lambda_{i} \int_{0}^{\infty} x_{i} \mathbb{E}_{\left\{\Phi_{j}\right\}} \prod_{l_{i}=1}^{S_{i}} \mathbb{P}\left\{\mathrm{SIR}_{x_{i}, l}^{\mathrm{MRC}} \geq \beta_{i} \mid\left\{\Phi_{j}\right\}\right\} d x_{i},
\end{gathered}
$$

where the first equation is according to Slivnyak- and Campbell-Mecke's Theorems [38]. We then note that conditioned to processes $\Phi_{j}$ s, the SIR values across streams are statistically independent. For a fixed value of $x_{i}$, we have

$$
\begin{aligned}
& \mathbb{P}\left\{\mathrm{SIR}_{x_{i}, l_{i}}^{\mathrm{MRC}} \geq \beta_{i} \mid\left\{\Phi_{j}\right\}\right\}=\mathbb{P}\left\{H_{x_{i}, l_{i}}^{\mathrm{MRC}} \geq \frac{\beta_{i}\left(\epsilon_{i}^{2} \tilde{H}_{x_{i}, l_{i}}^{\mathrm{MRC}}+\hat{H}_{x_{i}, l_{i}}^{\mathrm{MRC}}\right)}{1-\epsilon_{i}^{2}}+\frac{S_{i} \beta_{i} x_{i}^{\alpha}}{P_{i}\left(1-\epsilon_{i}^{2}\right)} \sum_{j \in \mathcal{K}} \sum_{x_{j} \in \Phi_{j} / x_{i}} \frac{P_{j}}{S_{j}}\left\|x_{j}\right\|^{-\alpha} G_{x_{j}, l_{i}}^{\mathrm{MRC}} \mid\left\{\Phi_{j}\right\}\right\} \\
& =\int_{0}^{\infty} \mathcal{L}_{\overline{\mathrm{F}}_{H_{i}}^{\mathrm{MRC}}}^{-1}\left(t_{i}\right) \prod_{j \in \mathcal{K}} \prod_{x_{j} \in \Phi_{j} / x_{i}} \mathbb{E}_{G_{x_{j}, l_{i}}^{\mathrm{MRC}}} e^{-t_{i} \frac{\beta_{i} S_{i} x_{i}^{\alpha}}{P_{i}\left(1-\epsilon_{i}^{2}\right)} \frac{P_{j}}{S_{j}}\left\|x_{j}\right\|^{-\alpha} G_{x_{j}, l_{i}}^{\mathrm{MRC}}} \mathbb{E} e^{-t_{i} \frac{\beta_{i}\left(\epsilon_{i}^{2} \tilde{H}_{x_{i}, l_{i}}^{\mathrm{MRC}}+\hat{H}_{x_{i}, l_{i}}^{\mathrm{MRC}}\right)}{1-\epsilon_{i}^{2}}} d t_{i} \\
& =\int_{0}^{\infty} \frac{\mathcal{L}_{\overline{\mathrm{F}}_{H_{i} \mathrm{MRC}}^{-1}}^{-1}\left(t_{i}\right)}{\left(1+\frac{t_{i} \beta_{i}}{1-\epsilon_{i}^{2}}\right)^{S_{i}-1}\left(1+\frac{t_{i} \epsilon_{i}^{2} \beta_{i}}{1-\epsilon_{i}^{2}}\right)} \prod_{j \in \mathcal{K}} \prod_{x_{j} \in \Phi_{j} / x_{i}} \mathbb{E}_{G_{x_{j}, l_{i}}^{\mathrm{MRC}}} e^{-t_{i} \frac{\beta_{i} S_{i} x_{i}^{\alpha}}{P_{i}\left(1-\epsilon_{i}^{2}\right)} \frac{P_{j}}{S_{j}\left\|x_{j}\right\| \alpha} G_{x_{j}, l_{i}}^{\mathrm{MRC}}} d t_{i},
\end{aligned}
$$

where $\mathcal{L}_{\overline{\mathrm{F}}_{H_{i}^{\mathrm{MRC}}}^{-1}}^{-1}\left(t_{i}\right)$ is the inverse Laplace transform of $H_{i}^{\mathrm{MRC}}, \mathcal{L}_{\overline{\mathrm{F}}_{H_{i}^{\mathrm{MRC}}}^{-1}}\left(t_{i}\right)=\sum_{m=0}^{N^{r}-1} \frac{1}{m !} \delta^{(m)}(t-1)$ (see, Lemmas 1 and 2 in [34]), and $\delta^{(m)}(t)$ is the $m$-th derivative of the Dirac delta function. Note that in (8) we drop index $l_{i}$ from $\mathcal{L}_{\overline{\mathrm{F}}_{H_{i}^{\mathrm{zf}}}}\left(t_{i}\right)$ because $H_{x_{i}, l_{i}}^{\mathrm{zf}}$ are identical random variables (rv.) across the streams. Substituting (8) into (7) followed by straightforward derivations yields

$$
\begin{aligned}
& \mathcal{P}_{C}^{\mathrm{MRC}}=\sum_{i \in \mathcal{K}} 2 \pi \lambda_{i} \int_{0}^{\infty} x_{i} \mathbb{E}_{\left\{\Phi_{j}\right\}} \prod_{l_{i}=1}^{S_{i}} \int_{0}^{\infty} \frac{\mathcal{L}_{\overline{\mathrm{F}}_{H_{i}^{\mathrm{MRC}}}^{-1}}\left(t_{i}\right) \prod_{j \in \mathcal{K}} \prod_{x_{j} \in \Phi_{j} / x_{i}} \mathbb{E}_{G_{x_{j}, l_{i}}^{\mathrm{MRC}}} e^{-t_{i} \frac{\beta_{i} S_{i} x_{i}^{\alpha}}{P_{i}\left(1-\epsilon_{i}^{2}\right)} \frac{P_{j}}{S_{j}}\left\|x_{j}\right\|^{-\alpha} G_{x_{j}, l_{i}}^{\mathrm{MRC}}} d t_{i} d x_{i}}{\left(1+\frac{t_{i} \beta_{i}}{1-\epsilon_{i}^{2}}\right)^{S_{i}-1}\left(1+\frac{t_{i} \epsilon_{i}^{2} \beta_{i}}{1-\epsilon_{i}^{2}}\right)} \\
& =\sum_{i \in \mathcal{K}} 2 \pi \lambda_{i} \int_{0}^{\infty} x_{i} d x_{i} \mathbb{E}_{\Phi} \int_{0}^{\infty} \ldots \int_{0}^{\infty} \prod_{j \in \mathcal{K}} \prod_{x_{j} \in \Phi_{j} / x_{i}} \mathbb{E}_{G_{x_{j}}^{\mathrm{MRC}}} \prod_{l_{i}=1}^{S_{i}} e^{-\frac{\beta_{i} S_{i} x_{i}^{\alpha}}{P_{i}\left(1-\epsilon_{i}^{2}\right)} \frac{P_{j} G_{x_{j}, l_{i} t_{l_{i}}}^{\mathrm{MRC}}}{S_{j}\left\|x_{j}\right\| \alpha}} \prod_{l_{i}=1}^{S_{i}} \frac{\mathcal{L}_{\overline{\mathrm{F}}_{H_{i}}^{\mathrm{MRC}}}^{-1}\left(t_{l_{i}}\right) d t_{l_{i}}}{\left(1+\frac{t_{l_{i}} \beta_{i}}{1-\epsilon_{i}^{2}}\right)^{S_{i}-1}\left(1+\frac{t_{l_{i}} \epsilon_{i}^{2} \beta_{i}}{1-\epsilon_{i}^{2}}\right)},
\end{aligned}
$$

as rv.s $G_{x_{j}, l_{i}}^{\mathrm{zf}}$ are independent and identically distributed (i.i.d.) rv.s across the streams. Thus,

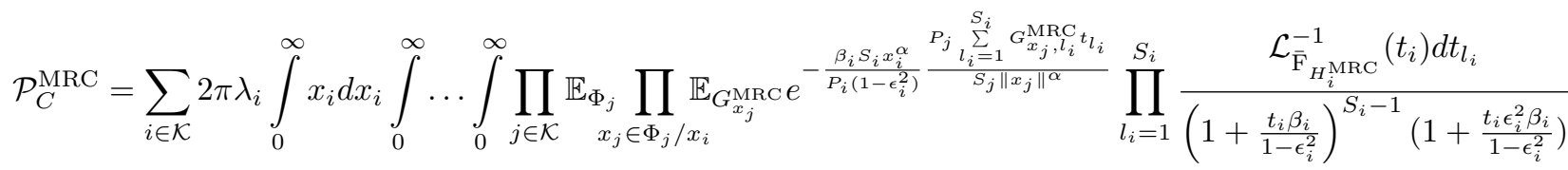

$$
\begin{aligned}
& =\sum_{i \in \mathcal{K}} 2 \pi \lambda_{i} \int_{0}^{\infty} x_{i} d x_{i} \int_{0}^{\infty} \ldots \int_{0}^{\infty} \prod_{l_{i}=1}^{S_{i}} \frac{e^{-\frac{x_{i}^{2}}{S_{i}} \tilde{C}(\alpha)\left(\frac{\beta_{i} S_{i}}{P_{i}\left(1-\epsilon_{i}^{2}\right)}\right)^{\check{\alpha}} \sum_{j=1}^{K} \lambda_{j}\left(\frac{P_{j}}{S_{j}}\right)^{\check{\alpha}} \mathbb{E}_{G_{j}^{\mathrm{MRC}}}\left[\left(\sum_{l_{i}=1}^{S_{i}} G_{j, l_{i}}^{\mathrm{MRC}} t_{l_{i}}\right)^{\check{\alpha}}\right]} \mathcal{L}_{\overline{\mathrm{F}}_{H_{i}^{\mathrm{MRC}}}}\left(t_{i}\right) d t_{l_{i}}}{\left(1+\frac{t_{i} \beta_{i}}{1-\epsilon_{i}^{2}}\right)^{S_{i}-1}\left(1+\frac{t_{i} \epsilon_{i}^{2} \beta_{i}}{1-\epsilon_{i}^{2}}\right)} .
\end{aligned}
$$


Reordering the integrals we then have

$$
\begin{aligned}
& \mathcal{P}_{C}^{\mathrm{MRC}}=\sum_{i \in \mathcal{K}} 2 \pi \lambda_{i} \int_{0}^{\infty} \ldots \int_{0}^{\infty} \prod_{l_{i}=1}^{S_{i}} \frac{\mathcal{L}_{\overline{\mathrm{F}}_{H_{i}^{\mathrm{MRC}}}^{-1}}\left(t_{i}\right) d t_{l_{i}}}{\left(1+\frac{t_{i} \beta_{i}}{1-\epsilon_{i}^{2}}\right)^{S_{i}-1}\left(1+\frac{t_{i} \epsilon_{i}^{2} \beta_{i}}{1-\epsilon_{i}^{2}}\right)} \int_{0}^{\infty} x_{i} e^{-x_{i}^{2} \tilde{C}(\alpha)\left(\frac{\beta_{i} S_{i}}{P_{i}\left(1-\epsilon_{i}^{2}\right)}\right)^{\check{\alpha}} \sum_{j=1}^{K} \lambda_{j}\left(\frac{P_{j}}{S_{j}}\right)^{\check{\alpha}} \mathbb{E}\left[\left(\sum_{l_{i}=1}^{S_{i}} G_{j, l_{i}}^{\mathrm{MRC}} t_{l_{i}}\right)^{\check{\alpha}}\right]} d x_{i}
\end{aligned}
$$

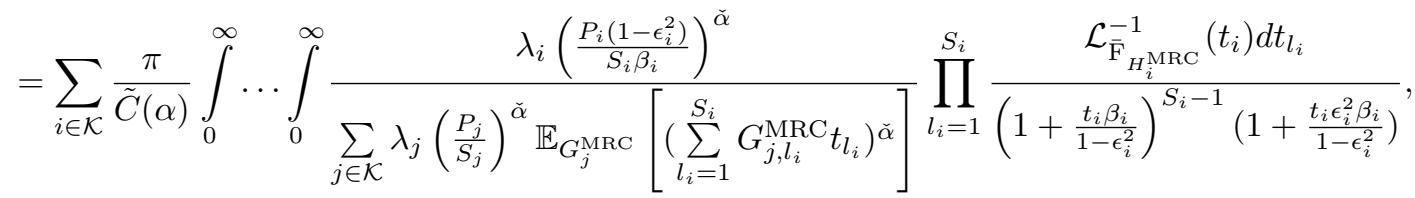

which is not easily tractable due to $\mathbb{E}_{G_{j}^{\mathrm{MRC}}}\left[\left(\sum_{l_{i}=1}^{S_{i}} G_{j, l_{i}}^{\mathrm{MRC}} t_{l_{i}}\right)^{\check{\alpha}}\right]$. To make the analysis tractable, we transform $\sum_{l_{i}=1}^{S_{i}} G_{j, l_{i}}^{\mathrm{MRC}} t_{l_{i}}$ into a multiplicative form $\prod_{l_{i}=1}^{S_{i}} G_{j, l_{i}}^{\mathrm{MRC}} t_{l_{i}}$ so that expectation operation on $G_{j, l_{i}}^{\mathrm{MRC}}$ becomes effective irrespective of variables $t_{l_{i}} \mathrm{~s}$. To do this, we adopt the arithmeticgeometric inequality, which results in the following upper-bound on the coverage probability:

$$
\begin{aligned}
\mathcal{P}_{C}^{\mathrm{MRC}} & \leq \sum_{i \in \mathcal{K}} \frac{\frac{\pi}{\tilde{C}(\alpha)}\left(\frac{P_{i}\left(1-\epsilon_{i}^{2}\right)}{S_{i} \beta_{i}}\right)^{\check{\alpha}} \frac{\lambda_{i}}{S_{i}^{\check{\alpha}}}}{\sum_{j=1}^{K} \lambda_{j}\left(\frac{P_{j}}{S_{j}}\right)^{\check{\alpha}} \mathbb{E}_{G_{j}^{\mathrm{MRC}}} \prod_{l_{i}=1}^{S_{i}}\left(G_{j, l_{i}}^{\mathrm{MRC}}\right)^{\frac{\check{\alpha}}{S_{i}}}} \ldots \int_{0}^{\infty} \prod_{0}^{S_{i}} \frac{t_{l_{i}}^{-\frac{\check{\alpha}}{S_{i}}} \mathcal{L}_{\overline{\mathrm{F}}_{H_{i} \mathrm{MRC}}}^{-1}\left(t_{l_{i}}\right)}{\left(1+\frac{t_{i} \beta_{i}}{1-\epsilon_{i}^{2}}\right)^{S_{i}-1}\left(1+\frac{t_{i} \epsilon_{i}^{2} \beta_{i}}{1-\epsilon_{i}^{2}}\right)} d t_{l_{i}} \\
& =\sum_{i \in \mathcal{K}} \frac{\frac{\pi}{\tilde{C}(\alpha)}\left(\frac{P_{i}\left(1-\epsilon_{i}^{2}\right)}{S_{i} \beta_{i}}\right)^{\check{\alpha}} \frac{\lambda_{i}}{S_{i}^{\check{\alpha}}}}{\sum_{j=1}^{K} \lambda_{j}\left(\frac{P_{j}}{S_{j}}\right)^{\check{\alpha}}\left(\mathbb{E}_{\left.G_{j}^{\mathrm{MRC}}\left(G_{j}^{\mathrm{MRC}}\right)^{\frac{\check{\alpha}}{S_{i}}}\right)^{S_{i}}}\right.}\left(\int_{0}^{S_{i}} \frac{t_{i}^{-\frac{\check{\alpha}}{S_{i}}} \mathcal{L}_{\overline{\mathrm{F}}_{H_{i} \mathrm{MRC}}^{-1}}\left(t_{i}\right)}{\left(1+\frac{t_{i} \beta_{i}}{1-\epsilon_{i}^{2}}\right)^{S_{i}-1}\left(1+\frac{t_{i} \epsilon_{i}^{2} \beta_{i}}{1-\epsilon_{i}^{2}}\right)}\right)^{,},
\end{aligned}
$$

where the last step is due to the fact that rv.s $G_{x_{j}, l_{i}}^{\mathrm{MRC}}$ are i.i.d. across streams. The integral in (9) is evaluated in Appendix A. The proof is done using the result of Appendix A, and noticing that $G_{x_{j}, l_{i}}^{\mathrm{MRC}}$ is chi-squared with $2 S_{j}$ DoFs.

Despite significant model complexities, Proposition 1 provides a closed-form upper-bound for the coverage probability. It is difficult to quantify the accuracy of the derived upper-bound as $G_{j, l_{i}}^{\mathrm{MRC}} \mathrm{s}$ are random in nature and $t_{l_{i}} \mathrm{~s}$ are integral variables. However, our simulation results in Section V indicate that the upper-bound of Proposition 1 is accurate and representative.

The bound on the coverage probability in (5) shows the effects of many important parameters such as the BS deployment density in each tier, their TX power and multiplexing gain, CSI inaccuracies, and the corresponding tiers' SIR threshold. The impact of the number of receive antennas is captured via parameter $\Theta\left(\beta_{i}, \epsilon_{i}, S_{i}\right)$ in (6). Note that the numerator and denominator of (5) correspond to the intended communication link, and the ICI, respectively.

A close examination of (5) in Proposition 1 provides significant insights on important design aspects of HetNets which are discussed in the following subsections. 

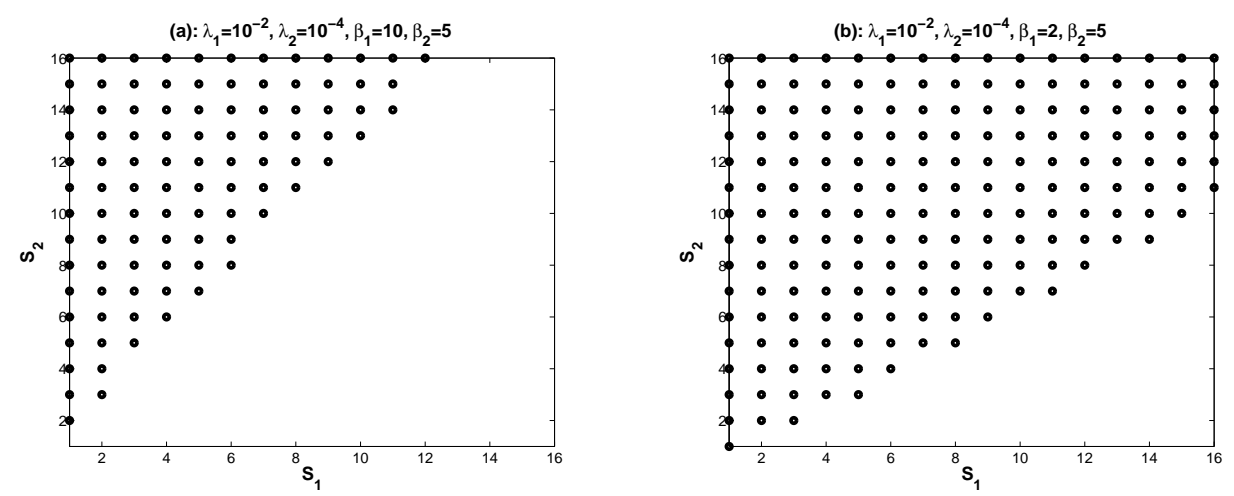

Fig. 1. Combinations of multiplexing gains for which $\frac{\partial \mathcal{P}_{\mathrm{C}}^{\mathrm{MRC}}}{\partial \tilde{\lambda}_{1}}>0$, where $\alpha=4, \epsilon_{1}=\epsilon_{2}=0.1, P_{1}=50 \mathrm{~W}$, and $P_{2}=1 \mathrm{~W}$.

\section{Does Densification Always Improve the Coverage Probability?}

We investigate the impact of densification on the coverage probability. We are interested in combinations of system parameters for which the coverage probability is increased by increasing the density of the BS in a given tier, namely tier $1: \frac{\partial \mathcal{P}_{C}^{\mathrm{MRC}}}{\partial \tilde{\lambda}_{1}}>0$. For brevity, we set $K=2$, and $\tilde{\lambda}_{1}=\lambda_{1}\left(P_{1} / S_{1}\right)^{\check{\alpha}}, \tilde{\lambda}_{2}=\lambda_{2}\left(P_{2} / S_{2}\right)^{\check{\alpha}}, A_{j i}=\left(\frac{\Gamma\left(\frac{\check{\alpha}}{S_{i}}+S_{j}\right)}{\Gamma\left(S_{j}\right)}\right)^{S_{i}}$. In this case, it can be shown that for $\frac{\partial \mathcal{P}_{C}^{\mathrm{MRC}}}{\partial \tilde{\lambda}_{1}}>0$, it is necessary to have $\frac{\tilde{\lambda}_{2}}{\tilde{\lambda}_{1}}\left(A_{21}-B A_{22}\right)<A_{12} B-A_{11}$, where $B=$ $\sqrt{\left(\frac{\left(1-\epsilon_{1}^{2}\right) \beta_{2} S_{2}}{\left(1-\epsilon_{2}^{2}\right) \beta_{1} S_{1}}\right)^{\check{\alpha}} \frac{\left(\Theta\left(\beta_{1}, \epsilon_{1}, S_{1}\right)\right)^{S_{1}} A_{21}}{\left(\Theta\left(\beta_{2}, \epsilon_{2}, S_{2}\right)\right)^{S_{2}} A_{12}}}$. Fig. 1 shows various combinations of the multiplexing gains that guarantee $\frac{\tilde{\lambda}_{2}}{\tilde{\lambda}_{1}}\left(A_{21}-B A_{22}\right)<A_{12} B-A_{11}$. In general, for densification of tier 1 to be effective in improving coverage performance, we need $S_{2}>S_{1}$. In fact, as decoding $S_{2}$ data streams is more unlikely than $S_{1}$ data streams, densification of tier 1 allows UEs to be more frequently be associated with tier 1 , thus improving the coverage probability. Moreover, by increasing $\beta_{1}$, we get a smaller number of multiplexing gain combinations, $\left(S_{1}, S_{2}\right)$, in which densification improves the coverage probability.

\section{Coverage Performance of Relevant MIMO Communications Scenarios}

Although Proposition 1 considers an open-loop tranceiver, one can utilize Proposition 1 to evaluate the coverage probability for various closed-loop scenarios, such as SISO $\left(N_{i}^{t}=N^{r}=1\right.$, $\forall i)$ [6], MISO-SDMA $\left(N^{r}=1\right)$ [12,28], Limited-feedback MISO-SDMA [28], and SIMO $\left(S_{i}=\right.$ $1, \forall i$ ). This is simply because the corresponding post-processing SIRs in the aforementioned closed loop techniques are often a function of the obtained SIR in (3). 
Assuming perfect CSI, immediate extensions of Proposition 1 are for zero-forcing beamforming $(\mathrm{ZFBF})$ at the receiver, and orthogonal space-time block codes (OSTBC). Such extensions can be done after making proper adjustments to the number of DoFs in the desired and interfering signals through the general framework proposed in [32].

\section{E. Selecting the Tranceiver Technique}

We compare two prevalent open-loop techniques: ZFBF and MRC. Here we assume a perfect CSIR, i.e., $\epsilon_{i}=0 \forall i$. We then set $\Theta^{\mathrm{ZF}}\left(S_{i}\right) \triangleq \sum_{m_{i}=0}^{N^{r}-S_{i}} \frac{\Gamma\left(\frac{\check{\alpha}}{S_{i}}+m_{i}\right)}{\Gamma\left(\frac{\alpha}{S_{i}}\right) \Gamma\left(1+m_{i}\right)}$. The coverage probability of the system with ZFBF was derived in [34] as:

$$
\mathcal{P}_{C}^{\mathrm{ZF}} \leq \frac{\pi}{\tilde{C}(\alpha)} \sum_{i \in \mathcal{K}} \frac{\lambda_{i}\left(\frac{P_{i}}{S_{i}^{2} \beta_{i}}\right)^{\check{\alpha}}\left(\Theta^{\mathrm{ZF}}\left(S_{i}\right)\right)^{S_{i}}}{\sum_{j \in \mathcal{K}} \lambda_{j}\left(\frac{P_{j}}{S_{j}}\right)^{\check{\alpha}}\left(\frac{\Gamma\left(\frac{\check{\alpha}}{S_{i}}+S_{j}\right)}{\Gamma\left(S_{j}\right)}\right)^{S_{i}}} .
$$

This is consistent with Proposition 1 , as $\mathcal{P}_{C}^{Z F}$ in (10) can also be obtained using the bound on $\mathcal{P}_{C}^{\mathrm{MRC}}$ in Proposition 1 , simply by substituting $\Theta\left(\beta_{i}, 0, S_{i}\right)$ in (5) with $\Theta^{\mathrm{ZF}}\left(S_{i}\right)$.

Using (10) and Proposition 1, we can now inspect whether ZFBF outperforms MRC. For clarity, we set $K=1$. It is then straightforward to confirm that $\mathcal{P}_{C}^{\mathrm{ZF}}>\mathcal{P}_{C}^{\mathrm{MRC}}$ if $\Theta^{\mathrm{ZF}}\left(S_{i}\right)>$ $\Theta\left(\beta_{i}, 0, S_{i}\right)$. Fig. 2 shows that, in general, ZFBF yields a higher coverage probability than MRC. This is mainly because the MRC receivers suffer from inter-stream interference. Furthermore, as shown in Fig. 2.a, by increasing the multiplexing gain, ZFBF becomes even more efficient than MRC. For a larger $N^{r}$, the superiority of ZFBF over MRC is shown to be reduced because the MRC receivers can harness diversity more effectively than ZFBF. Noticing that the ZFBF receiver complexity of a large arrays can be very high (because of the required matrix inversion operation), MRC provides room for compromising coverage performance (in fact, slightly for larger arrays) over computational complexity. Such aspects can be exploited in the design of HetNets. For instance, it is plausible to adaptively select either ZFBF or MRC in order to keep the prescribed coverage performance intact, while minimizing the complexity and energy consumption of the signal processing modules at the receivers.

Fig. 2.b also indicates that for a larger SIR threshold, $\beta$, ZFBF significantly outperforms MRC, while for small to moderate values of $\beta$, ZFBF is only slightly better than MRC. This observation suggests that for low-rate scenarios (e.g., for the cell-edge UEs) one can trade off 

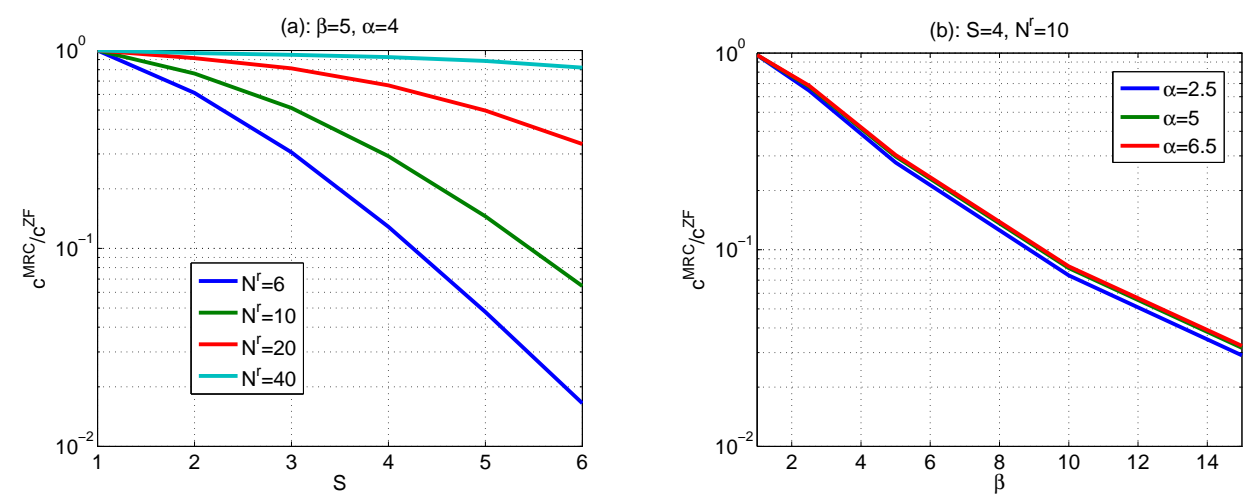

Fig. 2. (a): $\frac{\mathcal{P}_{C}^{\mathrm{MRC}}}{\mathcal{P}_{C}^{\mathrm{ZF}}}$, vs. the multiplexing gain $S$; (b): $\frac{\mathcal{P}_{C}^{\mathrm{MRC}}}{\mathcal{P}_{C}^{\mathrm{ZF}}}$ vs. the SIR threshold $\beta$.

a slightly higher performance for a significantly lower computational complexity. Fig. 2 further indicates that the relative performance of $\mathrm{ZFBF}$ and $\mathrm{MRC}$ is not related to the path-loss exponent.

\section{Cross-Stream SIR CorRelation}

As it is also shown in (7), for a given MIMO receiver, the SIR values across streams are statistically correlated mainly because of the correlated interference among antennas due to the common locations of interferers. More specifically, the interference originated from near-by BSs may cause a high level of interference simultaneously to all of the data streams transmitted to a typical UE. As shown in the proof of Proposition 1, the cross-stream SIR correlation renders analytical complexities. In this section, we characterize the aforementioned correlation and analyze its impact on the system coverage performance.

\section{A. SIR Correlation Coefficient}

In a link, the coverage probability is related to the joint SIRs' CDF of the streams. Here we focus on the SIR correlation instead of the ICI correlation. To quantify the SIR correlation, the Pearson correlation coefficients is used:

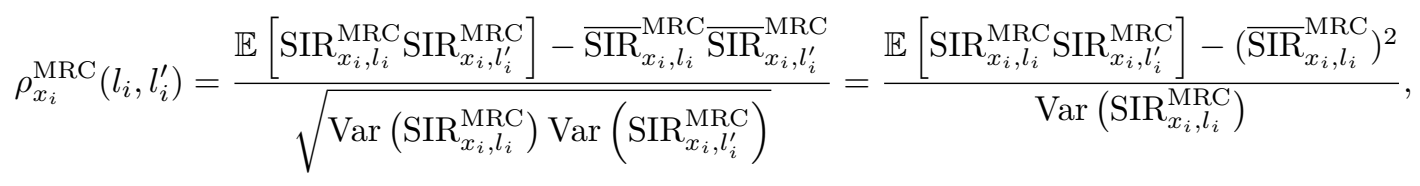

where $\mathbb{E}[$.$] is the expectation operator, \overline{\mathrm{SIR}}_{x_{i}, l_{i}}^{\mathrm{MRC}}$ is the average SIR value on data stream $l_{i}$, and $\operatorname{Var}[$.$] is the variance operator. The focus in the related literature (e.g., [35,48])$ is often on 


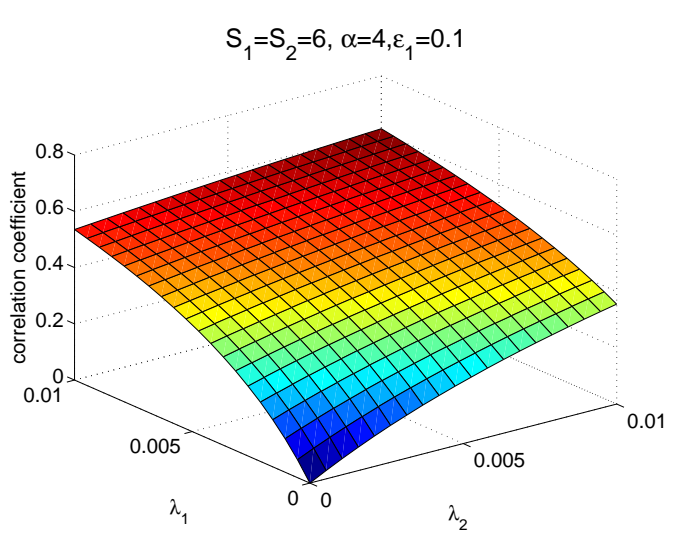

Fig. 3. Correlation coefficient vs. $\lambda_{1}$ and $\lambda_{2}$, where $K=2, N^{r}=8, x_{i}=20, P_{1}=50 \mathrm{~W}$, and $P_{2}=10 \mathrm{~W}$.

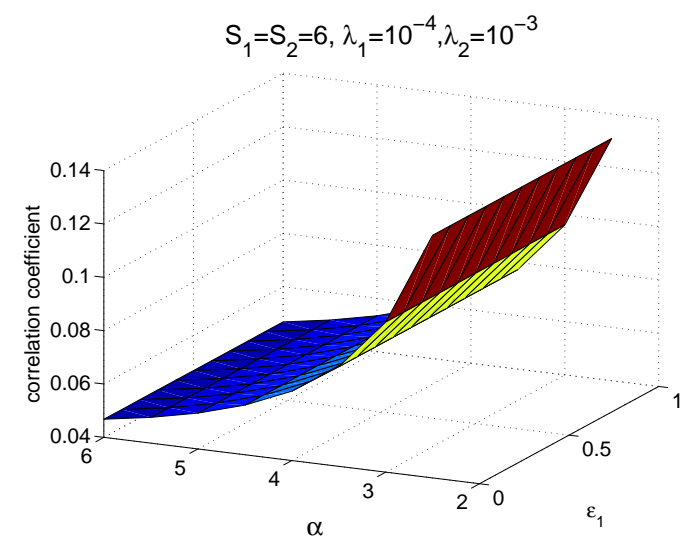

Fig. 4. Correlation coefficient vs. $\alpha$ and $\epsilon_{1}$.

understanding of the interference correlation among antennas. In contrast, as [49] we here focus on the SIR correlation among data streams.

Proposition 2: For the typical UE receiving data from BS, $x_{i}$, in a MIMO-MRC multiplexing system, the correlation coefficient between data streams $l_{i}$ and $l_{i}^{\prime}, \forall l_{i}, l_{i}^{\prime}, l_{i} \neq l_{i}^{\prime}$ is:

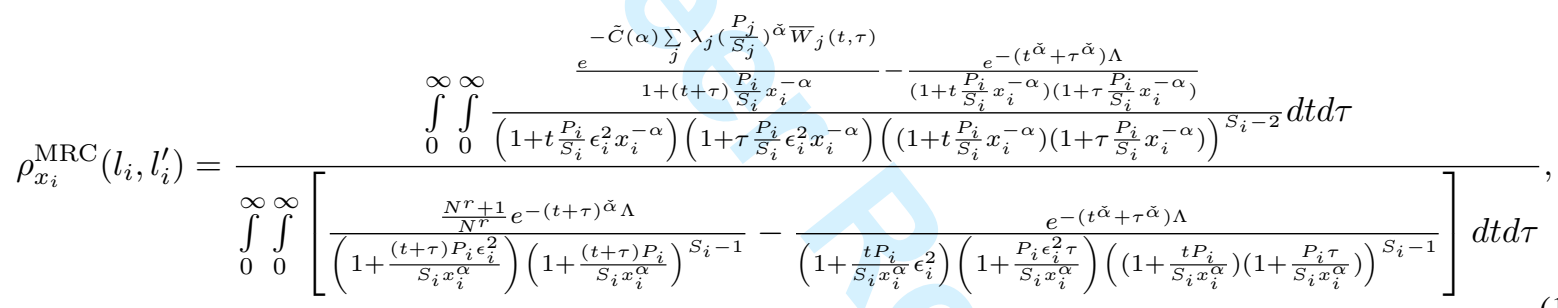

where $\check{\alpha}=2 / \alpha, \Lambda \triangleq \tilde{C}(\alpha) \sum_{j} \lambda_{j}\left(\frac{P_{j}}{S_{j}}\right)^{\check{\alpha}} \frac{\Gamma\left(\check{\alpha}+S_{j}\right)}{\Gamma\left(S_{j}\right)}, \tilde{C}(\alpha) \triangleq \pi \Gamma(1-\check{\alpha}), \Gamma(a) \triangleq \int_{0}^{\infty} e^{-z} z^{a-1} d z$, and

$$
\bar{W}_{j}(t, \tau) \triangleq \int_{0}^{\infty} \int_{0}^{\infty}\left(t g_{1}+\tau g_{2}\right)^{\check{\alpha}} \frac{\left(g_{1} g_{2}\right)^{S_{j}-1}}{\Gamma^{2}\left(S_{j}\right)} e^{-\left(g_{1}+g_{2}\right)} d g_{1} d g_{2} .
$$

Proof: See Appendix B.

As shown in (12), the ICI affects the correlation coefficient mainly through $\Lambda$, where $\Lambda$ is a function of BSs' density, their transmission powers and multiplexing gains, and the corresponding path-loss exponent. It is further shown in (12) that the multiplexing gains and CSI estimation inaccuracy may affect the correlation by imposing self-interference.

Fig. 3 shows the impact of $\lambda_{1}$ and $\lambda_{2}$ on $\rho_{x_{i}}^{\mathrm{MRC}}\left(l_{i}, l_{i}^{\prime}\right)$. As it is seen for a sparse network, where $\lambda_{1} \rightarrow 0$ and $\lambda_{2} \rightarrow 0$, the correlation coefficient is very close to 0 . In other words, the network behaves like an isolated link, where BSs are sparse in the coverage area. By increasing the 
density of BSs, however, $\rho_{x_{i}}^{\mathrm{MRC}}\left(l_{i}, l_{i}^{\prime}\right)$ is proportionally increased such that in an extreme case of high density of BSs where $\lambda_{1} \approx 0.01$ and/or $\lambda_{2} \approx 0.01$, the SIRs of data streams become highly correlated. In such a case, if a data stream, $l_{i}$, experiences outage due to a close-by interfering $\mathrm{BS}$, then other data streams $l_{i}^{\prime} \neq l_{i}$ will most likely experience the same.

Proposition 2 further shows that the imposed correlation due to the CSI estimation error seems negligible. This is because each individual data stream receives $S_{i}-1$ inter-stream interference which is much more powerful than the interference imposed by the CSI estimation error. Fig. 4 confirms this, indicating that the SIR correlation is not affected by change in the value of $\epsilon_{1}$.

The impact of path-loss exponent is also seen in Fig. 4. For a lower $\alpha$, even a small number of moderately close interferers induce a substantial level of interference. This reduces the SIR for all data streams at the same time, thus causing a large correlation among data streams. For a higher value of $\alpha$, the collective impact of the ICI received from the BSs located far from the receiver causes correlation, and hence unless the density of interferers is very high, the correlation is negligible.

One can therefore conclude that densification in multi-stream systems causes substantial SIR correlation among data streams through the ICI. This consequently affects the outage performance of the HetNet. Proposition 2, however, does not explicitly quantify the impact of the SIR correlation on the coverage performance.

\section{B. Impact of SIR Correlation on the Coverage Performance}

To analyze the impact of cross-stream SIR correlation on the coverage performance, here we introduce a multiplexing setting, namely full-correlation (FC) where the interference is fully correlated across all data streams in a link ${ }^{2}$. In other words, in the FC setting, the same level of ICI is received among all data streams in the communication link. Therefore, exchanging $G_{x_{j}, l_{i}}^{\mathrm{MRC}}$ with its average value, $S_{j}$, the ICI in the FC setting is $I^{\mathrm{FC}}=\sum_{j \in \mathcal{K}} \sum_{x_{j} \in \Phi_{j} / x_{i}} P_{j}\left\|x_{j}\right\|^{-\alpha}$. Assuming a typical UE is associated with BS $x_{i}$, the corresponding post-processing SIR for stream $l_{i}$ is

$$
\mathrm{SIR}_{x_{i}, l_{i}}^{\mathrm{MRC}-\mathrm{FC}}=\frac{\frac{P_{i}}{S_{i}}\left\|x_{i}\right\|^{-\alpha}\left(1-\epsilon_{i}^{2}\right) H_{x_{i}, l_{i}}^{\mathrm{MRC}}}{\frac{P_{i}}{S_{i}\left\|x_{i}\right\|^{\alpha}}\left(\hat{H}_{x_{i}, l_{i}}^{\mathrm{MRC}}+\epsilon_{i}^{2} \tilde{H}_{x_{i}, l_{i}}^{\mathrm{MRC}}\right)+I^{\mathrm{FC}}} .
$$

\footnotetext{
${ }^{2}$ In [36] a similar assumption made to quantify signal correlation of optimal-combining in SIMO ad hoc networks.
} 
Based on the adopted CA policy, the associated BS for a link is the one that its corresponding smallest SIR values $\mathrm{SIR}_{x_{i}, l_{i}}^{\mathrm{MRC}-\mathrm{FC}}$ across all data streams, is the maximum among all the BSs. Therefore, the typical UE is in coverage if

$$
\mathcal{A}_{\text {all }}^{\mathrm{MRC}-\mathrm{FC}}=\left\{\exists i \in \mathcal{K}: \max _{x_{i} \in \Phi_{i} l_{i}=1, \ldots, S_{i}} \operatorname{SIR}_{x_{i}, l_{i}}^{\mathrm{MRC}-\mathrm{FC}} \geq \beta_{i}\right\}
$$

is not empty. An upper-bound on the corresponding coverage probability, $\mathcal{P}_{C}^{\mathrm{MRC}-\mathrm{FC}}$, is given in the following proposition.

Proposition 3: In the FC setting, the coverage probability is upper-bounded as:

$$
\mathcal{P}_{C}^{\mathrm{MRC}-\mathrm{FC}} \leq \frac{\pi}{\tilde{C}(\alpha) \sum_{j=1}^{K} \lambda_{j} P_{j}^{\check{\alpha}}} \sum_{i \in \mathcal{K}} \lambda_{i}\left(\frac{P_{i}\left(1-\epsilon_{i}^{2}\right)}{S_{i}^{2} \beta_{i}}\right)^{\check{\alpha}}\left(\Theta\left(\beta_{i}, \epsilon_{i}, S_{i}\right)\right)^{S_{i}} .
$$

Proof: We prove the proposition by following the same line of argument as in the proof of Proposition 1. In the FC setting, (7) is reduced to

$$
\begin{aligned}
& \mathcal{P}_{C}^{\mathrm{MRC}-\mathrm{FC}}=\sum_{i \in \mathcal{K}} 2 \pi \lambda_{i} \int_{0}^{\infty} x_{i} \mathbb{E}_{\left\{I^{\mathrm{FC}}\right\}} \prod_{l_{i}=1}^{S_{i}} \mathbb{P}\left\{\mathrm{SIR}_{x_{i}, l}^{\mathrm{MRC}-\mathrm{FC}} \geq \beta_{i} \mid I^{\mathrm{FC}}\right\} d x_{i}
\end{aligned}
$$

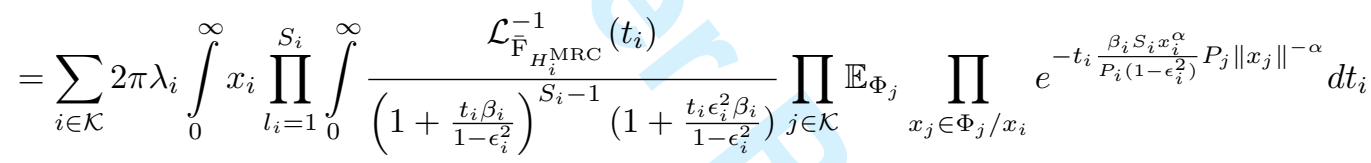

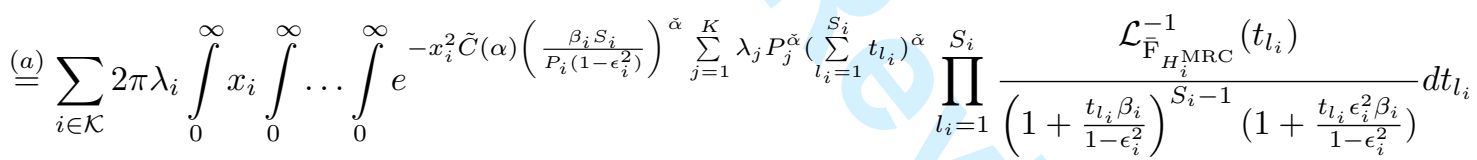

$$
\begin{aligned}
& \stackrel{(b)}{=} \frac{\pi}{\tilde{C}(\alpha) \sum_{j=1}^{K} \lambda_{j} P_{j}^{\check{\alpha}}} \sum_{i \in \mathcal{K}} \lambda_{i}\left(\frac{\beta_{i} S_{i}}{P_{i}\left(1-\epsilon_{i}^{2}\right)}\right)^{-\check{\alpha}} \int_{0}^{\infty} \ldots \int_{0}^{\infty}\left(\sum_{l_{i}=1}^{S_{i}} t_{l_{i}}\right)^{-\check{\alpha}} \prod_{l_{i}=1}^{S_{i}} \frac{\mathcal{L}_{\overline{\mathrm{F}}_{H_{i} \mathrm{MRC}}}^{-1}\left(t_{l_{i}}\right)}{\left(1+\frac{t_{l_{i}} \beta_{i}}{1-\epsilon_{i}^{2}}\right)^{S_{i}-1}\left(1+\frac{t_{l_{i}} \epsilon_{i}^{2} \beta_{i}}{1-\epsilon_{i}^{2}}\right)} d t_{l_{i}} \\
& \stackrel{(c)}{\leq} \frac{\pi}{\tilde{C}(\alpha) \sum_{j=1}^{K} \lambda_{j} P_{j}^{\check{\alpha}}} \sum_{i \in \mathcal{K}} \lambda_{i}\left(\frac{\beta_{i} S_{i}^{2}}{P_{i}\left(1-\epsilon_{i}^{2}\right)}\right)^{-\check{\alpha}} \int_{0}^{\infty} \ldots \int_{0}^{\infty} \prod_{l_{i}=1}^{S_{i}} \frac{t_{l_{i}}^{-\frac{\check{\alpha}}{S_{i}}} \mathcal{L}_{\overline{\mathrm{F}}_{H_{i}^{\mathrm{MRC}}}^{-1}}^{\left(t_{l_{i}}\right)}}{\left(1+\frac{t_{l_{l} \beta_{i}}}{1-\epsilon_{i}^{2}}\right)^{S_{i}-1}\left(1+\frac{t_{l_{i}} \epsilon_{i}^{2} \beta_{i}}{1-\epsilon_{i}^{2}}\right)} d t_{l_{i}} \\
& \stackrel{(d)}{=} \frac{\pi}{\tilde{C}(\alpha) \sum_{j=1}^{K} \lambda_{j} P_{j}^{\check{\alpha}}} \sum_{i \in \mathcal{K}} \lambda_{i}\left(\frac{\beta_{i} S_{i}^{2}}{P_{i}\left(1-\epsilon_{i}^{2}\right)}\right)^{-\check{\alpha}} \prod_{l_{i}=1}^{S_{i}} \int_{0}^{\infty} \frac{t_{l_{i}}^{-\frac{\check{\alpha}}{S_{i}}} \mathcal{L}_{\overline{\mathrm{F}}_{H_{i}^{\mathrm{MRC}}}^{-1}}^{-1}\left(t_{l_{i}}\right)}{\left(1+\frac{t_{l_{i}} \beta_{i}}{1-\epsilon_{i}^{2}}\right)^{S_{i}-1}\left(1+\frac{t_{l_{i}} \epsilon_{i} \beta_{i}}{1-\epsilon_{i}^{2}}\right)} d t_{l_{i}} \\
& \stackrel{(e)}{=} \frac{\pi}{\tilde{C}(\alpha) \sum_{j=1}^{K} \lambda_{j} P_{j}^{\check{\alpha}}} \sum_{i \in \mathcal{K}} \lambda_{i}\left(\frac{\beta_{i} S_{i}^{2}}{P_{i}\left(1-\epsilon_{i}^{2}\right)}\right)^{-\check{\alpha}}\left(\int_{0}^{\infty} \frac{t_{i}^{-\frac{\check{\alpha}}{S_{i}}} \mathcal{L}_{\overline{\mathrm{F}}_{H_{i}^{\mathrm{MRC}}}^{-1}}^{-1}\left(t_{i}\right)}{\left(1+\frac{t_{i} \beta_{i}}{1-\epsilon_{i}^{2}}\right)^{S_{i}-1}\left(1+\frac{t_{i} \epsilon_{i}^{2} \beta_{i}}{1-\epsilon_{i}^{2}}\right)} d t_{i}\right)^{S_{i}}
\end{aligned}
$$


where in (a) we insert the Laplace transform of $I^{\mathrm{FC}}$ and in (b) the integrals are reordered and we integrate the inner integral with respect to $x_{i}$. In (c) arithmetic-geometric inequality is applied followed by (d) and (e) where the fading gains, $H_{x_{i}, l_{i}}^{\mathrm{MRC}}$, are i.i.d. Applying the result of Appendix A in (e), completes the proof.

Comparing Propositions 1 and 3, we note that in general for the FC setting, the coverage probability has a more simplified form. On the other hand, the upper-bound of the coverage performance of a MIMO-MRC HetNet system is (almost) always higher than the same system assuming the FC setting. This is because by noting that for $\frac{\check{\alpha}}{S_{i}} \in(0,1)$, there holds $\frac{\Gamma\left(\frac{\check{\alpha}}{S_{i}}+S_{j}\right)}{\Gamma\left(S_{j}\right)} \lesssim S_{j}^{\frac{\check{\alpha}}{S_{i}}}$ [35]. Therefore, noticing that both (16) and (5) have the same nominator while the denominator of the former is larger than that of the latter, we obtain $\mathcal{P}_{C}^{\mathrm{MRC}-\mathrm{FC}} \lesssim \mathcal{P}_{C}^{\mathrm{MRC}}$. Consequently, we can conclude that adding to the correlation among data streams of a communication link can reduce the coverage probability. Although this result is based on the derived upper-bounds on the coverage probabilities in (16) and (5), our simulation results in Section V confirm its credibility.

\section{What If the Cross-Stream SIR Correlation Is Overlooked?}

The above analysis shows that approximating a practical scenario based on the FC setting results in underestimation of the coverage probability. Another way to simplify the coverage analysis is to simply ignore the cross stream SIR correlation, i.e., statistically independent SIR values. We refer to this case as no-correlation (NC) setting. Starting from (7) and assuming the $\mathrm{NC}$ setting, the coverage probability in (7) is written as

$$
\mathcal{P}_{C}^{\mathrm{MRC}-\mathrm{NC}}=\sum_{i \in \mathcal{K}} 2 \pi \lambda_{i} \int_{0}^{\infty} x_{i} \prod_{l_{i}=1}^{S_{i}} \mathbb{E}_{\Phi} \mathbb{P}\left\{\operatorname{SIR}_{x_{i}, l_{i}}^{\mathrm{MRC}} \geq \beta_{i} \mid \Phi\right\} d x_{i} .
$$

The coverage probability in (18) can then be written as:

$$
\begin{aligned}
& \mathcal{P}_{C}^{\mathrm{MRC}-\mathrm{NC}}=\sum_{i \in \mathcal{K}} 2 \pi \lambda_{i} \int_{0}^{\infty} x_{i} \prod_{l_{i}=1}^{S_{i}} \int_{0}^{\infty} \frac{\mathcal{L}_{\overline{\mathrm{F}}_{H_{i}^{\mathrm{MRC}}}^{-1}}\left(t_{i}\right)}{\left(1+\frac{t_{i} \beta_{i}}{1-\epsilon_{i}^{2}}\right)^{S_{i}-1}\left(1+\frac{t_{i} \epsilon_{i}^{2} \beta_{i}}{1-\epsilon_{i}^{2}}\right)} \prod_{j \in \mathcal{K}} \mathbb{E}_{\Phi_{j}} \prod_{x_{j} \in \Phi_{j} / x_{i}} \mathbb{E}_{G_{x_{j}, l_{i}}^{\mathrm{MRC}}} e^{-t_{l_{i}} \frac{\beta_{i} S_{i} x_{i}^{\alpha}}{P_{i}\left(1-\epsilon_{i}^{2}\right)} \frac{P_{j} G_{x_{j}, l_{i}}^{\mathrm{MRC}}}{S_{j}\left\|x_{j}\right\|^{\alpha}}} d t_{i} \\
& \stackrel{(a)}{=} \sum_{i \in \mathcal{K}} 2 \pi \lambda_{i} \int_{0}^{\infty} x_{i} \int_{0}^{\infty} \ldots \int_{0}^{\infty} e^{-x_{i}^{2} \Lambda\left(\frac{\beta_{i} S_{i}}{P_{i}\left(1-\epsilon_{i}^{2}\right)}\right)^{\check{\alpha}}\left(\sum_{l_{i}=1}^{S_{i}} t_{l_{i}}^{\check{\alpha}}\right)} \prod_{l_{i}=1}^{S_{i}} \frac{\mathcal{L}_{\overline{\mathrm{F}}_{H_{i}^{\mathrm{MRC}}}^{-1}}^{-1}\left(t_{l_{i}}\right)}{\left(1+\frac{t_{l_{i}} \beta_{i}}{1-\epsilon_{i}^{2}}\right)^{S_{i}-1}\left(1+\frac{t_{l_{i}} \epsilon_{i}^{2} \beta_{i}}{1-\epsilon_{i}^{2}}\right)} d t_{l_{i}}
\end{aligned}
$$

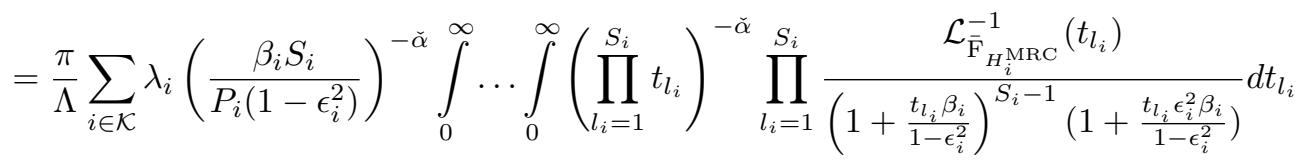




$$
\begin{gathered}
=\frac{\pi}{\Lambda} \sum_{i \in \mathcal{K}} \lambda_{i}\left(\frac{\beta_{i} S_{i}^{2}}{P_{i}\left(1-\epsilon_{i}^{2}\right)}\right)^{-\check{\alpha}} \prod_{l_{i}=1}^{S_{i}} \int_{0}^{\infty} \frac{t_{l_{i}}^{-\check{\alpha}} \mathcal{L}_{\overline{\mathrm{F}}_{H_{i}^{\mathrm{MRC}}}^{-1}}\left(t_{l_{i}}\right)}{\left(1+\frac{t_{l_{i}} \beta_{i}}{1-\epsilon_{i}^{2}}\right)^{S_{i}-1}\left(1+\frac{t_{l_{i}} \epsilon_{i}^{2} \beta_{i}}{1-\epsilon_{i}^{2}}\right)} d t_{l_{i}} \\
\stackrel{(b)}{=} \frac{\pi}{\Lambda} \sum_{i \in \mathcal{K}} \lambda_{i}\left(\frac{\beta_{i} S_{i}^{2}}{P_{i}\left(1-\epsilon_{i}^{2}\right)}\right)^{-\check{\alpha}}\left(\int_{0}^{\infty} \frac{t_{i}^{-\check{\alpha}} \mathcal{L}_{\overline{\mathrm{F}}_{H_{i}}^{\mathrm{MRC}}}^{-1}\left(t_{i}\right)}{\left(1+\frac{t_{i} \beta_{i}}{1-\epsilon_{i}^{2}}\right)^{S_{i}-1}\left(1+\frac{t_{i} \epsilon_{i}^{2} \beta_{i}}{1-\epsilon_{i}^{2}}\right)} d t_{i}\right)^{S_{i}},
\end{gathered}
$$

where in (a) we insert the Laplace transform of the ICI and further notice the definition of $\Lambda$ as in Proposition 2. Denoting the integral in (b) by $\tilde{\Theta}\left(\beta_{i}, \epsilon_{i}, S_{i}\right)$ and following the same line of argument as in Appendix A, we evaluate this integral as

$$
\tilde{\Theta}\left(\beta_{i}, \epsilon_{i}, S_{i}\right) \triangleq \sum_{r_{i}=0}^{N^{r}-1} \sum_{q_{i}=0}^{r_{i}} \sum_{p_{i}=0}^{q_{i}} \frac{(-1)^{q_{i}-p_{i}} \beta_{i}^{2 q_{i}-p_{i}}}{\epsilon_{i}^{-4 q_{i}+2 p_{i}}\left(1-\epsilon_{i}^{2}\right)^{S_{i}}} \frac{\left(1-\epsilon_{i}^{2}+\beta_{i}\right)^{-q_{i}-S_{i}+1}\left(1+\epsilon_{i}^{2}\left(\beta_{i}-1\right)\right)^{-q_{i}+p_{i}-1}}{p_{i} \mathrm{~B}\left(S_{i}-1, p_{i}\right)\left(r_{i}-q_{i}\right) \mathrm{B}\left(\check{\alpha}, r_{i}-q_{i}\right)} .
$$

Using this, (18) is then reduced to

$$
\mathcal{P}_{C}^{\mathrm{MRC}-\mathrm{NC}}=\frac{\pi}{\Lambda} \sum_{i \in \mathcal{K}} \lambda_{i}\left(\frac{P_{i}\left(1-\epsilon_{i}^{2}\right)}{S_{i} \beta_{i}}\right)^{\check{\alpha}}\left(\tilde{\Theta}\left(\beta_{i}, \epsilon_{i}, S_{i}\right)\right)^{S_{i}} .
$$

Note that $\mathrm{NC}$ setting is in fact an extreme case and thus $\mathcal{P}_{C}^{\mathrm{MRC}-\mathrm{NC}}$ is not practically achievable. This is simply because it does not comply with the max-SIR CA rule as in the NC setting, an independent set of interferers appears on each data stream. Therefore, there might be cases where the typical UE becomes associated with different BSs for different data streams. This, however, contradicts the reality of the MIMO signal model as presented in 1.

We further note that, as $\check{\alpha} \in(0,1)$, by using $\frac{\Gamma\left(\check{\alpha}+S_{j}\right)}{\Gamma\left(S_{j}\right)} \lesssim S_{j}^{\check{\alpha}}$ a lower-bound on $\mathcal{P}_{C}^{\mathrm{MRC}-\mathrm{NC}}$ is

$$
\mathcal{P}_{C}^{\mathrm{MRC}-\mathrm{NC}} \gtrsim \frac{\pi \sum_{i \in \mathcal{K}} \lambda_{i}\left(\frac{P_{i}\left(1-\epsilon_{i}^{2}\right)}{S_{i}^{2} \beta_{i}}\right)^{\check{\alpha}}\left(\tilde{\Theta}\left(\beta_{i}, \epsilon_{i}, S_{i}\right)\right)^{S_{i}}}{\tilde{C}(\alpha) \sum_{j=1}^{K} \lambda_{j} P_{j}^{\check{\alpha}}} \geq \frac{\pi \sum_{i \in \mathcal{K}} \lambda_{i}\left(\frac{P_{i}\left(1-\epsilon_{i}^{2}\right)}{S_{i}^{2} \beta_{i}}\right)^{\check{\alpha}}\left(\Theta\left(\beta_{i}, \epsilon_{i}, S_{i}\right)\right)^{S_{i}}}{\tilde{C}(\alpha) \sum_{j=1}^{K} \lambda_{j} P_{j}^{\check{\alpha}}}=\mathcal{P}_{C}^{\mathrm{MRC}-\mathrm{FC}} .
$$

where the second inequality is because $\tilde{\Theta}\left(\beta_{i}, \epsilon_{i}, S_{i}\right) \geq \Theta\left(\beta_{i}, \epsilon_{i}, S_{i}\right)$. To confirm this, we notice that the beta function is a decreasing function of its argument, and observing that by comparing $\tilde{\Theta}\left(\beta_{i}, \epsilon_{i}, S_{i}\right)$ in (19) and $\Theta\left(\beta_{i}, \epsilon_{i}, S_{i}\right)$ in (6), we note that for a given positive number $a$, $\tilde{\Theta}\left(\beta_{i}, \epsilon_{i}, S_{i}\right)-\Theta\left(\beta_{i}, \epsilon_{i}, S_{i}\right) \propto \frac{1}{\mathrm{~B}(\check{\alpha}, a)}-\frac{1}{\mathrm{~B}\left(\frac{\check{\alpha}}{S_{i}}, a\right)}$.

On the other hand, since $\frac{\check{\alpha}}{S_{i}} \in(0,1)$, there holds $\frac{\Gamma\left(\frac{\check{\alpha}}{S_{i}}+S_{j}\right)}{\Gamma\left(S_{j}\right)}>S_{j}^{\frac{\check{\alpha}}{S_{i}}} \Gamma\left(1+\frac{\check{\alpha}}{S_{i}}\right)$ [35]. Applying this, $\mathcal{P}_{C}^{\mathrm{MRC}}$ in (5) is further upper-bounded as

$$
\mathcal{P}_{C}^{\mathrm{MRC}} \leq \frac{\pi \sum_{i \in \mathcal{K}} \lambda_{i}\left(\frac{P_{i}\left(1-\epsilon_{i}^{2}\right)}{S_{i}^{2} \beta_{i}}\right)^{\check{\alpha}}\left(\Theta\left(\beta_{i}, \epsilon_{i}, S_{i}\right) \Gamma\left(1+\frac{\check{\alpha}}{S_{i}}\right)\right)^{S_{i}}}{\tilde{C}(\alpha) \sum_{j=1}^{K} \lambda_{j} P_{j}^{\check{\alpha}}} \leq \frac{\left.\pi \sum_{i \in \mathcal{K}} \lambda_{i}\left(\frac{P_{i}\left(1-\epsilon_{i}^{2}\right)}{S_{i}^{2} \beta_{i}}\right)^{\check{\alpha}}\left(\Theta\left(\beta_{i}, \epsilon_{i}, S_{i}\right)\right)\right)^{S_{i}}}{\tilde{C}(\alpha) \sum_{j=1}^{K} \lambda_{j} P_{j}^{\check{\alpha}}} \leq \mathcal{P}_{C}^{\mathrm{MRC}-\mathrm{NC}},
$$


where the last line is because $\Gamma\left(1+\frac{\check{\alpha}}{S_{i}}\right) \leq 1$ for $\frac{\check{\alpha}}{S_{i}} \in(0,1)$. Consequently, using the NC setting, the coverage probability is basically overestimated. This implies that the common approach that focuses on either isolated scenarios or non-isolated scenarios but with emphasis of the characterization of MIMO communications from the perspective of a data stream is essentially overestimation of the actual performance of the network.

\section{Simulation Results}

In this section, we use simulations to evaluate the performance of a MIMO-MRC HetNet setting and further examine the accuracy of the developed analysis. The simulated system is a 2-tier HetNet, i.e., $K=2$. The macro BS in the first tier has a high Tx power of $P_{1}=50 \mathrm{~W}$. The second tier consists of femto BSs with a low Tx power of $P_{2}=1 \mathrm{~W}$. The path-loss exponent is $\alpha=4$, and the CSI estimation error $\epsilon_{i}=0.1 \forall i, N_{1}^{t}=N_{2}^{t}=16$. In a disk with radius 10,000 units, we randomly drop BSs of each tier according to the corresponding tier densities. We set $\lambda_{U}=1$ so all the BSs are assumed to be active. We apply Monte Carlo technique and analyze 40, 000 snapshots of simulations. In each snapshot the MIMO channels are randomly generated. For the UEs, the corresponding SIR values are then calculated based on the MRC receiver.

\section{A. Impact of Path-loss Exponent, CSI Estimation Error, and SIR Threshold}

Fig. 5.a shows the coverage probability $v$. the estimation error, $\epsilon=\epsilon_{i}, \forall i$, for several values of the path-loss exponent, $\alpha$. The bound obtained in Prop. 1 is shown to be close to the simulation result. Also, increasing the CSI inaccuracy is shown to reduce the coverage performance. This is because the interference on each data stream is increased due to the CSI inaccuracy. It is also seen in Fig. 5.a that increasing the path-loss exponent improves the outage performance. Noting that a larger $\alpha$ implies a smaller signal strength, the improved outage performance suggests that the ICI is the main limiting factor.

Fig. 5.a also shows that in contrast to the cases with a smaller path-loss exponent (e.g., outdoor communications), the coverage is not significantly affected by the CSI inaccuracy where the path-loss exponent is high (e.g., indoor communications). This suggests that a simpler transceiver 

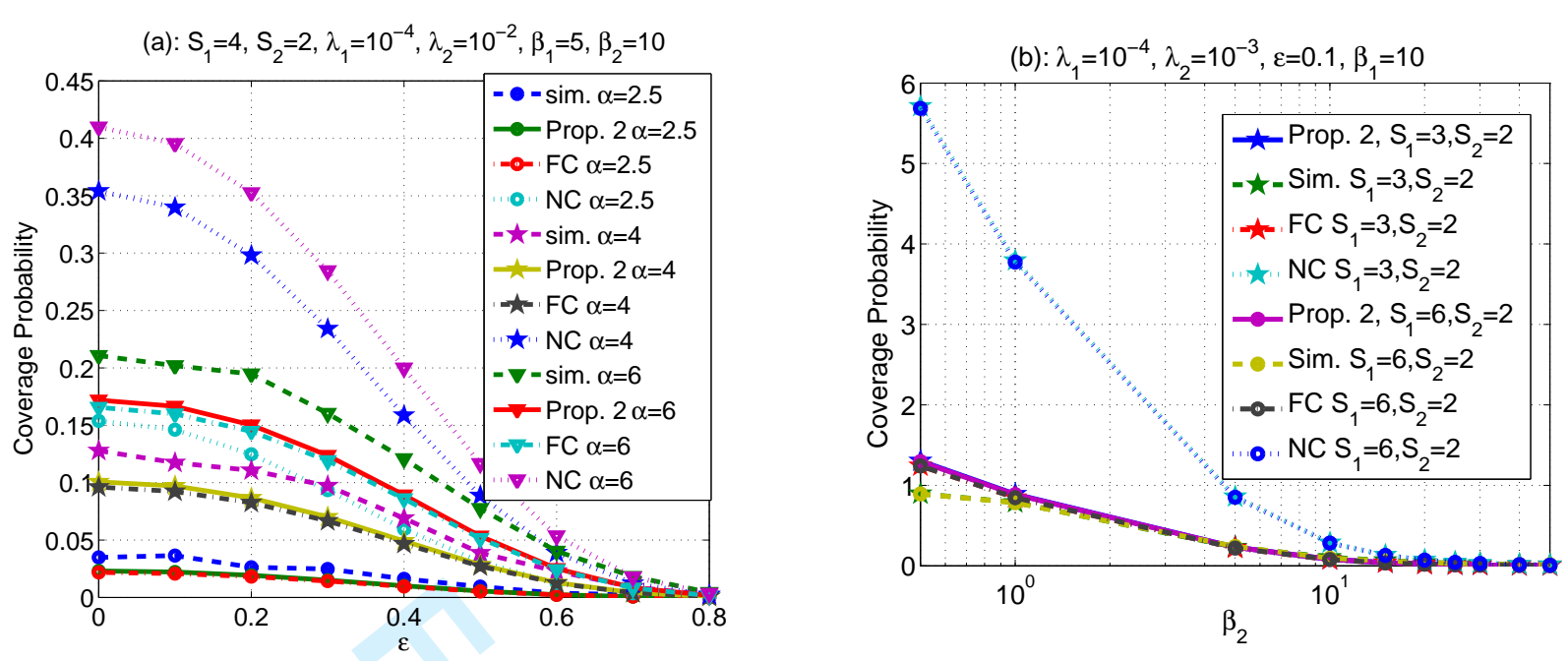

Fig. 5. (a): Coverage Probability vs. the CSI estimation error. (b): Coverage Probability vs. $\beta_{2}$.

design or/and signaling protocol can be used without any significant compromise of the coverage probability. Fig. 5.b shows the coverage probability versus $\beta_{2}$. The bound obtained in Prop. 1 is is shown to be sufficiently accurate even for small values of $\beta_{2}$. It also shows that a higher $\beta_{2}$ results in a lower coverage performance.

\section{B. Impact of Densificaiton and Multiplexing Gains}

In Figs. 6 and 7 the coverage probability is given versus $\lambda_{1}$. We consider 5 settings (Stg) of multiplexing gains between two tiers, where Stg1, Stg2, Stg3, Stg4, and Stg5, respectively, refer to $\left(S_{1}=1, S_{2}=1\right),\left(S_{1}=4, S_{2}=1\right),\left(S_{1}=4, S_{2}=2\right),\left(S_{1}=1, S_{2}=2\right)$, and $\left(S_{1}=8, S_{2}=2\right)$. Fig. 6 shows the coverage performance for Stg1, Stg2, and Stg3. The results of Stg1, Stg4, and Stg5 are plotted in Fig. 7. Both figures show the outage performance for $\lambda_{2}=10^{-3}$, and $\lambda_{2}=10^{-2}$.

It is seen in Figs. 6 and 7 that the analytical result presented in Prop. 1 closely follows the simulation results. It is also observed that a single stream communications, Stg1, generally outperforms the other combinations of multiplexing gains, regardless of the density of the BSs in both tiers. For the single stream case, it is also seen that densification in tier 1 always results in a higher improvement in the coverage probability. Nevertheless, comparison of Fig. 6.a with 
Fig. 6.b (resp. Fig. 7.a with Fig. 7.b) suggests that the improvement of the coverage probability by increasing $\lambda_{1}$ is reduced if tier 2 is also densified at the same time.

Fig. 6 also shows that for a small to moderate $\lambda_{1}$, increasing $S_{1}$ from 1 to $4(\operatorname{Stg} 1 \rightarrow \operatorname{Stg} 2)$ does not compromise coverage performance. However, for a sufficiently large $\lambda_{1}$, the coverage performance in Stg2 is significantly reduced. Comparing Fig. 6.a with Fig. 6.b, we further observe that for a higher value of $\lambda_{2}$, the positive impact of having a larger $S_{1}$ on the coverage performance is lower. Therefore, densification in tier 2 allows the growth of multiplexing gain in tier 1. This is because for a larger $\lambda_{2}$, the UEs are more likely to be associated with the BSs in tier 2. This is because the successful decoding of a data streams where $S_{2}=1$ is more probable than that of $S_{1}=4$, so the coverage probability is improved.

Results in Fig. 7 show that for a small to moderate $\lambda_{1}$, increasing $S_{2}$ from 1 to 2 (Stg1 $\rightarrow$ Stg4) substantially reduces the coverage performance. To tackle this problem, one may consider increasing $\lambda_{1}$ which reduces the performance gap. For a very dense tier 1, the coverage performance of Stg1 and Stg4 are then converged. Comparing Fig. 7.a with Fig. 7.b, one can see that by increasing $\lambda_{2}$, the impact of $S_{2}$ on the coverage performance is increased. Therefore, when densifying tier 2, increasing its multiplexing gain is not recommended. This is because for a larger $\lambda_{2}$, the UEs are more likely to be associated with the BSs in tier 2. The chance of successful decoding of $S_{2}=2$ is less than that of $S_{2}=1$, and hence the coverage probability is reduced. To address this issue, one might densify tier 1. By increasing $\lambda_{1}$, UEs are more often associated with the BSs in tier 1 , where $S_{1}=1$ and it is more likely for the data stream to be successfully decoded.

It is further seen in Figs. 6 and 7 that both Stg3 and Stg4 similarly perform with a low coverage performance, where densification neither in tier 1 nor in tier 2, can compensate the significant coverage reduction compared to Stg1. This is because in cases where both $S_{1}$ and $S_{2}$ are high, successful decoding of data streams is less likely, even for a high density of the BSs. For such cases, reducing the multiplexing gains seems the only way to improve the coverage performance. 
(a): $\lambda_{2}=10^{-3}$

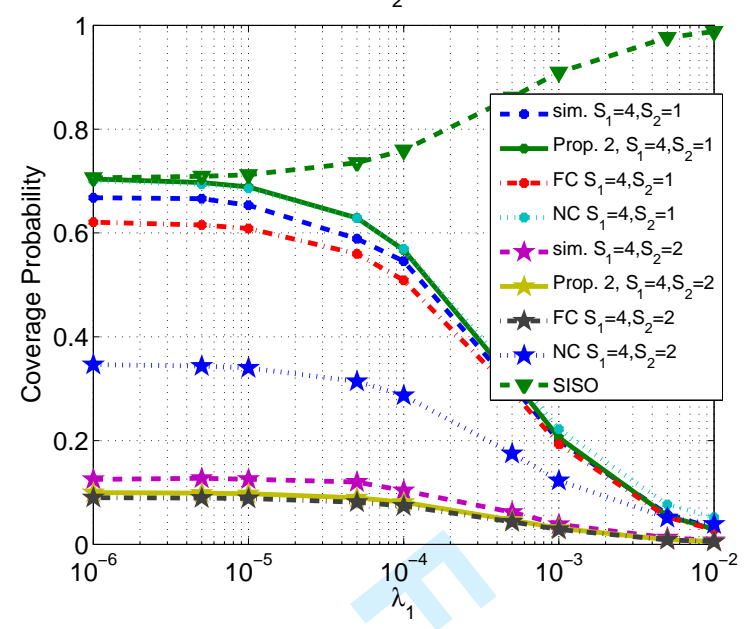

(b): $\lambda_{2}=10^{-2}$

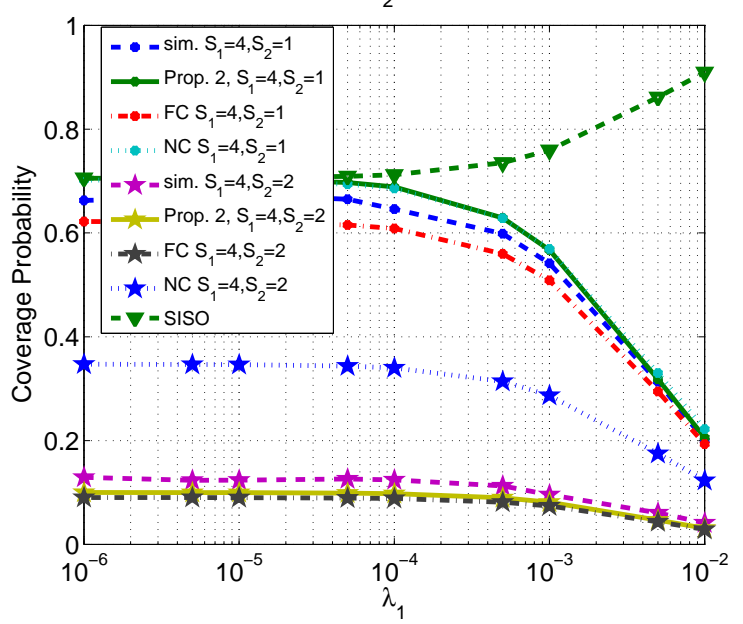

Fig. 6. (a): Coverage Probability vs. $\lambda_{1}$ when $\lambda_{2}=10^{-3}$; (b): Coverage Probability vs. $\lambda_{1}$ when $\lambda_{2}=10^{-2}$, where $\beta_{1}=5$, $\beta_{2}=10$, and $N^{r}=10$.

(a): $\lambda_{2}=10^{-3}$

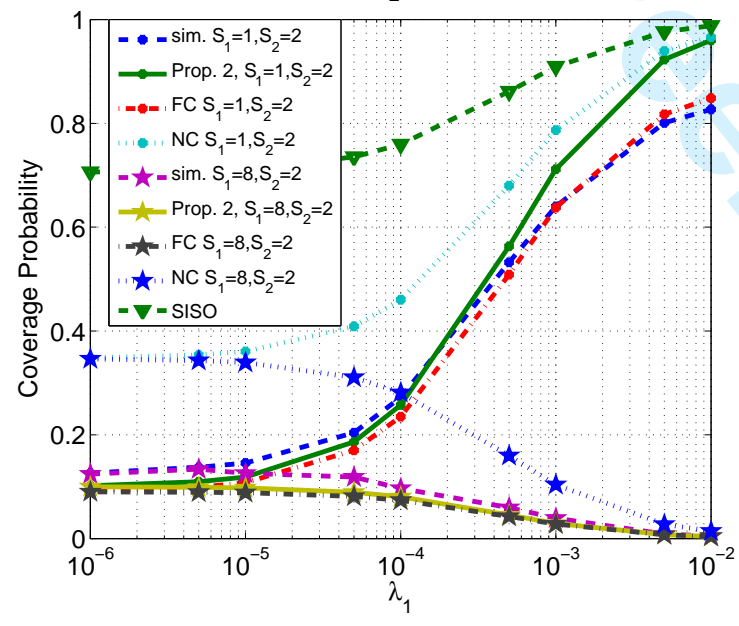

(b): $\lambda_{2}=10^{-2}$

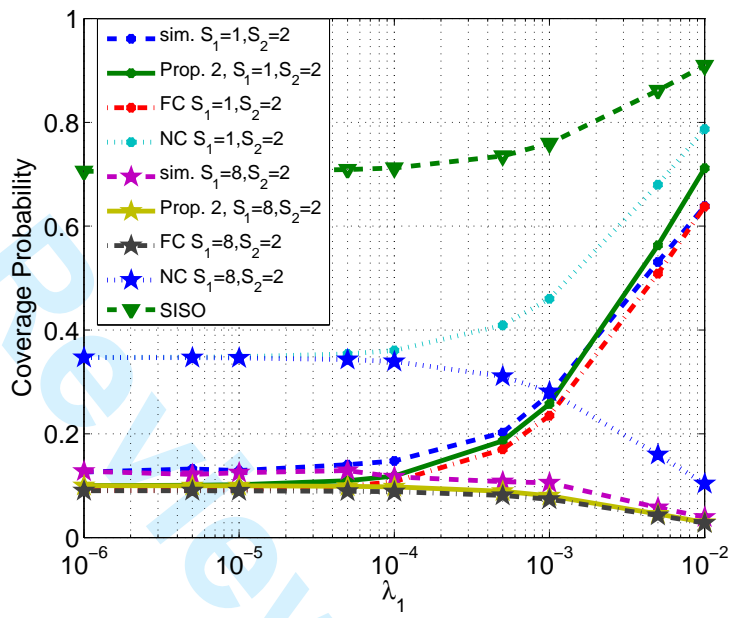

Fig. 7. (a): Coverage Probability vs. $\lambda_{1}$ when $\lambda_{2}=10^{-3}$; (b): Coverage Probability vs. $\lambda_{1}$ when $\lambda_{2}=10^{-2}$, where $\beta_{1}=5$, $\beta_{2}=10$, and $N^{r}=10$.

\section{Impact of the SIR Correlation}

In Section IV we quantitatively investigated the impact of SIR correlation on the coverage probability. We show that under FC setting the upper-bound of the coverage probability is underestimated, whereas by ignoring SIR correlation, the coverage probability is overestimated. These results in Figs 5, 6, and 7 confirmed the above analysis. 
We further observe that the coverage probability in the NC setting substantially overestimates the coverage performance, while surprisingly the FC setting slightly underestimates the coverage performance. From Fig. 5.a we also notice that for a smaller $\alpha$, the coverage probability in the FC setting becomes more accurate for the same reason as the observation made in Fig. 4, where a smaller path-loss exponent results in a larger SIR correlation.

\section{CONCLUSION}

Adopting tools of stochastic geometry, we studied the coverage probability of MIMO-MRC multiplexing systems in HetNets. Our analysis incorporated impacts of many important system parameters including the density of BSs, transmission powers, SIR thresholds, multiplexing gains, and CSI inaccuracies on the coverage performance. We derived an accurate upper-bound on the coverage probability in a closed-form. Important engineering insights were derived from scrutinizing our analytical and simulation results: $(i)$ densification in multiplexing systems will be practiced in conjunction with multiplexing gains, else dramatic coverage loss might be inevitable; (ii) in indoor scenarios (high path-loss exponent regimes) it is possible to reduce the pilot signaling overhead designated for CSI estimation without imposing noticeable coverage loss; (iii) although MRC suffers from intra-stream interference in comparison to more complex receivers such as ZFBF, the relative coverage loss in large array scenarios and/or for cell edge users is barely noticeable.

We also developed analytical tools facilitating thorough investigations of the impacts of crossstream SIR correlation on the coverage performance of multi-stream systems. Specifically, by focusing on the communication scenarios that the successful decoding of all transmitted data streams are required for the coverage, assuming full correlation among data streams is shown to yield a slightly smaller coverage performance. On the other hand, our analysis proved that by neglecting such correlation, as commonly assumed in the literature, one should expect a substantial overestimation of the coverage probability.

The results in this paper can be further utilized for performance bench-marking, where the performance of advanced MIMO techniques is compared to zero-feedback MRC. Such a com- 
parison provides quantitative insights on the cost versus the benefit of adopting such techniques, e.g., the higher computational complexities and the required signaling overheads versus the gain on the coverage. Besides, our results provide an analytical tool for designing system parameters and aspects such as the required CSI accuracy and network coverage performance based on the wireless environment characteristics, such as path-loss exponent.

\section{Appendix A: Closed-Form For (9)}

Using the characteristics of Dirac delta function, we write

$$
\begin{gathered}
\int_{0}^{\infty} \frac{t_{i}^{-\frac{\check{\alpha}}{S_{i}}} \mathcal{L}_{\overline{\mathrm{F}}_{H_{i}^{\mathrm{MRC}}}^{-1}}\left(t_{i}\right)}{\left(1+\frac{t_{i} \beta_{i}}{1-\epsilon_{i}^{2}}\right)^{S_{i}-1}\left(1+\frac{t_{i} \epsilon_{i}^{2} \beta_{i}}{1-\epsilon_{i}^{2}}\right)} d t_{i}=\sum_{r_{i}=0}^{N^{r}-1} \frac{1}{r_{i} !} \int_{0}^{\infty} \delta^{\left(r_{i}\right)}\left(t_{i}-1\right) \frac{t_{i}^{-\frac{\check{\alpha}}{S_{i}}}}{\left(1+\frac{t_{i} \beta_{i}}{1-\epsilon_{i}^{2}}\right)^{S_{i}-1}\left(1+\frac{t_{i} \epsilon_{i}^{2} \beta_{i}}{1-\epsilon_{i}^{2}}\right)} d t_{i} \\
=\left.\sum_{r_{i}=0}^{N^{r}-1} \frac{(-1)^{r_{i}}}{r_{i} !} \frac{d^{r_{i}}}{d t^{r_{i}}} \frac{t^{-\frac{\check{\alpha}}{S_{i}}}}{\left(1+\frac{t_{i} \beta_{i}}{1-\epsilon_{i}^{2}}\right)^{S_{i}-1}\left(1+\frac{t_{i} \epsilon_{i}^{2} \beta_{i}}{1-\epsilon_{i}^{2}}\right)}\right|_{t=1} .
\end{gathered}
$$

Applying the Leibniz rule along with straightforward mathematical derivation we then get

$$
\begin{gathered}
\frac{d^{r_{i}}}{d t^{r_{i}}} \frac{t^{-\frac{\check{\alpha}}{S_{i}}}}{\left(1+\frac{t \beta_{i}}{1-\epsilon_{i}^{2}}\right)^{S_{i}-1}\left(1+\frac{t \epsilon_{i}^{2} \beta_{i}}{1-\epsilon_{i}^{2}}\right)}=\sum_{q_{i}=0}^{r_{i}}\left(\begin{array}{c}
r_{i} \\
q_{i}
\end{array}\right) \frac{d^{q_{i}}}{d t^{q_{i}}} \frac{1}{\left(1+\frac{t \beta_{i}}{1-\epsilon_{i}^{2}}\right)^{S_{i}-1}\left(1+\frac{t \epsilon_{i}^{2} \beta_{i}}{1-\epsilon_{i}^{2}}\right)} \frac{d^{r_{i}-q_{i}}}{d t^{r_{i}-q_{i}}} t^{-\frac{\check{\alpha}}{S_{i}}} \\
=\sum_{q_{i}=0}^{r_{i}}\left(\begin{array}{c}
r_{i} \\
q_{i}
\end{array}\right) \sum_{p_{i}=0}^{q_{i}}\left(\begin{array}{c}
q_{i} \\
p_{i}
\end{array}\right) \frac{d^{p_{i}}}{d t^{p_{i}}} \frac{1}{\left(1+\frac{t \beta_{i}}{1-\epsilon_{i}^{2}}\right)^{S_{i}-1}} \frac{d^{q_{i}-p_{i}}}{d t^{q_{i}-p_{i}}} \frac{1}{\left(1+\frac{t \epsilon_{i}^{2} \beta_{i}}{1-\epsilon_{i}^{2}}\right)} \frac{d^{r_{i}-q_{i}}}{d t^{r_{i}-q_{i}}} t^{-\frac{\check{\alpha}}{S_{i}}}
\end{gathered}
$$

which is

$$
\begin{gathered}
(22)=\sum_{q_{i}=0}^{r_{i}} \sum_{p_{i}=0}^{q_{i}} \frac{\left(\begin{array}{c}
r_{i} \\
q_{i}
\end{array}\right)\left(\begin{array}{c}
q_{i} \\
p_{i}
\end{array}\right) \prod_{m_{i}=0}^{p_{i}-1}\left(S_{i}-1+m_{i}\right)}{\left(\frac{-\beta_{i}}{1-\epsilon_{i}^{2}}\right)^{-q_{i}}\left(1+\frac{t \beta_{i}}{1-\epsilon_{i}^{2}}\right)^{q_{i}+S_{i}-1} \frac{\prod_{u_{i}=0}^{q_{i}-p_{i}-1}\left(1+u_{i}\right)}{\left(\frac{-\beta_{i} \epsilon_{i}^{2}}{1-\epsilon_{i}^{2}}\right)^{-q_{i}+p_{i}}\left(1+\frac{t \beta_{i} \epsilon_{i}^{2}}{1-\epsilon_{i}^{2}}\right)^{q_{i}-p_{i}+1}} \frac{\prod_{n_{i}=0}^{r_{i}-q_{i}-1}\left(\frac{\check{\alpha}}{S_{i}}+n_{i}\right)}{(-1)^{-r_{i}+q_{i}} t^{\frac{\check{\alpha}}{S_{i}}+r_{i}-q_{i}}}} \\
=(-1)^{r_{i}} \sum_{q_{i}=0}^{r_{i}} \sum_{p_{i}=0}^{q_{i}} \frac{(-1)^{q_{i}-p_{i}}\left(\begin{array}{c}
r_{i} \\
q_{i}
\end{array}\right)\left(\begin{array}{l}
q_{i} \\
p_{i}
\end{array}\right) \Gamma\left(S_{i}-1+p_{i}\right) \Gamma\left(q_{i}-p_{i}+1\right) \Gamma\left(\frac{\check{\alpha}}{S_{i}}+r_{i}-q_{i}\right)}{\epsilon_{i}^{2 p_{i}-4 q_{i}}\left(1+\frac{\beta_{i}}{1-\epsilon_{i}^{2}}\right)^{q_{i}+S_{i}-1}\left(\frac{\beta_{i}}{1-\epsilon_{i}^{2}}\right)^{p_{i}-2 q_{i}}\left(1+\frac{\beta_{i} \epsilon_{i}^{2}}{1-\epsilon_{i}^{2}}\right)^{q_{i}-p_{i}+1} \Gamma\left(S_{i}-1\right) \Gamma\left(\frac{\check{\alpha}}{S_{i}}\right)} .
\end{gathered}
$$

Using this, (21) is then re written as

$$
(21)=\sum_{r_{i}=0}^{N^{r}-1} \sum_{q_{i}=0}^{r_{i}} \sum_{p_{i}=0}^{q_{i}} \frac{(-1)^{q_{i}-p_{i}} \beta_{i}^{2 q_{i}-p_{i}}}{\epsilon_{i}^{-4 q_{i}+2 p_{i}}} \frac{\left(1-\epsilon_{i}^{2}+\beta_{i}\right)^{-q_{i}-S_{i}+1}\left(1+\epsilon_{i}^{2}\left(\beta_{i}-1\right)\right)^{-q_{i}+p_{i}-1}}{\left(1-\epsilon_{i}^{2}\right)^{S_{i}} p_{i} \mathrm{~B}\left(S_{i}-1, p_{i}\right)\left(r_{i}-q_{i}\right) \mathrm{B}\left(\frac{\tilde{\alpha}}{S_{i}}, r_{i}-q_{i}\right)} .
$$




\section{APPENDIX B: ProOF OF PROPOSITION 2}

We start with the evaluation of $\overline{\mathrm{SIR}}_{x_{i}, l_{i}}^{\mathrm{MRC}}$. Due to the independence of the intended and interfering signals, and noting that $H_{x_{i}, l_{i}}^{\mathrm{MRC}}$ is a chi-square distributed with $2 N^{r}$ DoFs, we write

$$
\overline{\operatorname{SIR}}_{x_{i}, l_{i}}^{\mathrm{MRC}}=\frac{P_{i} N^{r}\left(1-\epsilon_{i}^{2}\right)}{S_{i} x_{i}^{\alpha}} \mathbb{E}\left(\frac{P_{i}}{S_{i} x_{i}^{\alpha}}\left(\epsilon_{i}^{2} \tilde{H}_{x_{i}, l_{i}}^{\mathrm{MRC}}+\hat{H}_{x_{i}, l_{i}}^{\mathrm{MRC}}\right)+\sum_{j \in \mathcal{K}} \sum_{x_{j} \in \Phi_{j} / x_{i}} \frac{P_{j} G_{x_{j}, l_{i}}^{\mathrm{MRC}}}{S_{j}\left\|x_{j}\right\|^{\alpha}}\right)^{-1} .
$$

Using $\int_{0}^{\infty} e^{-s z} d s=z^{-1}$, the expectation in (24) is evaluated as

$$
\begin{aligned}
& \mathbb{E} \int_{0}^{\infty} e^{-t \frac{P_{i}}{S_{i} x_{i}^{\alpha}}\left(\epsilon_{i}^{2} \tilde{H}_{x_{i}, l_{i}}^{\mathrm{MRC}}+\hat{H}_{x_{i}, l_{i}}^{\mathrm{MRC}}\right)-t \sum_{j \in \mathcal{K}} \sum_{x_{j} \in \Phi_{j} / x_{i}} \frac{P_{j} G_{x_{j}}^{\mathrm{MRC}, l_{i}}}{S_{j}\left\|x_{j}\right\|^{\alpha}}} d t \\
& \stackrel{(a)}{=} \int_{0}^{\infty} \mathbb{E} e^{-t \frac{P_{i}}{S_{i} x_{i}^{\alpha}} \epsilon_{i}^{2} \tilde{H}_{x_{i}, l_{i}}^{\mathrm{MRC}}} \mathbb{E} e^{-t \frac{P_{i}}{S_{i} x_{i}^{\alpha}} \hat{H}_{x_{i}, l_{i}}^{\mathrm{MRC}}} \mathbb{E}_{\left\{\Phi_{j}\right\}} \mathbb{E}_{\left\{G_{x_{j}, l_{i}}^{\mathrm{MRC}}\right\}_{x_{j}, \forall x_{j}}} \prod_{j \in \mathcal{K}} \prod_{x_{j} \in \Phi_{j} / x_{i}} e^{-t \frac{P_{j}}{S_{j}}\left\|x_{j}\right\|^{-\alpha} G_{x_{j}, l_{i}}^{\mathrm{MRC}}} d t \\
& \stackrel{(b)}{=} \int_{0}^{\infty} \frac{\prod_{j \in \mathcal{K}} \mathbb{E}_{\Phi_{j}} \prod_{x_{j} \in \Phi_{j} / x_{i}} \mathbb{E}_{G_{x_{j}, l_{i}}^{\mathrm{MRC}}} e^{-t \frac{P_{j}}{S_{j}}\left\|x_{j}\right\|^{-\alpha} G_{x_{j}, l_{i}}^{\mathrm{MRC}}}}{\left(1+t \frac{P_{i}}{S_{i} x_{i}^{\alpha}} \epsilon_{i}^{2}\right)\left(1+t \frac{P_{i}}{S_{i} x_{i}^{\alpha}}\right)^{S_{i}-1}} d t=\int_{0}^{\infty} \frac{e^{-t^{\check{\alpha}} \tilde{C}(\alpha) \sum_{j} \lambda_{j}\left(\frac{P_{j}}{S_{j}}\right)^{\check{\alpha}} \frac{\Gamma\left(\check{\alpha}+S_{j}\right)}{\Gamma\left(S_{j}\right)}}}{\left(1+t \frac{P_{i}}{S_{i} x_{i}^{\alpha}} \epsilon_{i}^{2}\right)\left(1+t \frac{P_{i}}{S_{i} x_{i}^{\alpha}}\right)^{S_{i}-1}} d t,
\end{aligned}
$$

where in (a) the independence of r.v.s is used, and in (b) we insert the Laplace transforms of r.v.s $\tilde{H}_{x_{i}, l_{i}}^{\mathrm{MRC}}$, which is a chi-square distributed r.v. with $2\left(S_{i}-1\right)$ DoFs, and $\tilde{H}_{x_{i}, l_{i}}^{\mathrm{MRC}}$, which is exponentially distributed, at point $\frac{P_{i}}{S_{i}} x_{i}^{-\alpha}$. In the last step, the following formula is used [50]:

$$
\mathbb{E}_{\Phi_{j}} \prod_{x_{j} \in \Phi_{j}} \mathbb{E}_{h_{x_{j}}} e^{-s_{j}\left\|x_{j}\right\|^{-\alpha} h_{x_{j}}}=e^{-\tilde{C}(\alpha) \lambda_{j} s_{j}^{\check{\alpha}} \mathbb{E}\left[h^{\check{\alpha}}\right]}
$$

in which $\mathbb{E}\left(G_{j}^{\mathrm{MRC}}\right)^{\check{\alpha}}=\frac{\Gamma\left(\check{\alpha}+S_{j}\right)}{\Gamma\left(S_{j}\right)}$ is substituted with $G_{j}^{\mathrm{MRC}}$ which is also a chi-squared r.v. with $2 S_{j}$ DoFs. Finally, substituting $\Lambda$ defined in Proposition 1 yields

$$
\overline{\operatorname{SIR}}_{x_{i}, l_{i}}^{\mathrm{MRC}}=\frac{P_{i} N^{r}\left(1-\epsilon_{i}^{2}\right)}{S_{i} x_{i}^{\alpha}} \int_{0}^{\infty} \frac{e^{-t^{\check{\alpha} \Lambda}}}{\left(1+t \frac{P_{i}}{S_{i} x_{i}^{\alpha}} \epsilon_{i}^{2}\right)\left(1+t \frac{P_{i}}{S_{i} x_{i}^{\alpha}}\right)^{S_{i}-1}} d t .
$$

To evaluate $\operatorname{Var}\left(\operatorname{SIR}_{x_{i}, l_{i}}^{\mathrm{svd}}\right)$, we need to evaluate $\mathbb{E}\left[\left(\operatorname{SIR}_{x_{i}, l_{i}}^{\mathrm{svd}}\right)^{2}\right]$ :

$$
\mathbb{E}\left[\left(\operatorname{SIR}_{x_{i}, l_{i}}^{\mathrm{MRC}}\right)^{2}\right]=\frac{\left(1-\epsilon_{i}^{2}\right)^{2} N^{r}\left(N^{r}+1\right)}{P_{i}^{-2} S_{i}^{2} x_{i}^{2 \alpha}} \int_{0}^{\infty} \int_{0}^{\infty} \frac{e^{-(t+\tau)^{\check{\alpha}} \Lambda}}{\left(1+(t+\tau) \frac{P_{i}}{S_{i} x_{i}^{\alpha}} \epsilon_{i}^{2}\right)\left(1+(t+\tau) \frac{P_{i}}{S_{i} x_{i}^{\alpha}}\right)^{S_{i}-1}} d t d \tau .
$$

Combining (26) and (27), $\operatorname{Var}\left(\operatorname{SIR}_{x_{i}, l_{i}}^{\mathrm{svd}}\right)$ is then obtained, and

$$
\mathbb{E}\left[\operatorname{SIR}_{x_{i}, l_{i}}^{\mathrm{MRC}} \operatorname{SIR}_{x_{i}, l_{i}^{\prime}}^{\mathrm{MRC}}\right]=\frac{\left(P_{i}\left(1-\epsilon_{i}^{2}\right) N^{r}\right)^{2}}{S_{i}^{2} x_{i}^{2 \alpha}} \mathcal{I}
$$


where

$$
\begin{gathered}
\mathcal{I}=\mathbb{E}\left(\frac{P_{i}}{S_{i} x_{i}^{\alpha}}\left(\epsilon_{i}^{2} \tilde{H}_{x_{i}, l_{i}}^{\mathrm{MRC}}+\hat{H}_{x_{i}, l_{i}}^{\mathrm{MRC}}\right)+\sum_{j \in \mathcal{K}} \sum_{x_{j} \in \Phi_{j} / x_{i}} \frac{P_{j} G_{x_{j}, l_{i}}^{\mathrm{MRC}}}{S_{j}\left\|x_{j}\right\|^{\alpha}}\right)^{-1}\left(\frac{P_{i}}{S_{i} x_{i}^{\alpha}}\left(\epsilon_{i}^{2} \tilde{H}_{x_{i}, l_{i}^{\prime}}^{\mathrm{MRC}}+\hat{H}_{x_{i}, l_{i}^{\prime}}^{\mathrm{MRC}}\right)+\sum_{j \in \mathcal{K}} \sum_{x_{j} \in \Phi_{j} / x_{i}} \frac{P_{j} G_{x_{j}, l_{i}^{\prime}}^{\mathrm{MRC}}}{S_{j}\left\|x_{j}\right\|^{\alpha}}\right)^{-1} . \\
=\int_{0}^{\infty} \int_{0}^{\infty} \frac{\mathbb{E} e^{-\sum_{j \in \mathcal{K} x_{j} \in \Phi_{j} / x_{i}} \frac{P_{j}}{S_{j}\left\|x_{j}\right\| \alpha}\left(t G_{x_{j}, l_{i}^{\prime}}^{\mathrm{MRC}+\tau G_{x_{j}, l_{i}^{\prime}}^{\mathrm{MRC}}}\right.} \mathbb{E} e^{-\frac{P_{i}}{S_{i} x_{i}^{\alpha}}\left(t \hat{H}_{x_{i}, l_{i}}^{\left.\mathrm{MRC}+\tau \hat{H}_{x_{i}, l_{i}^{\prime}}^{\mathrm{MRC}}\right)} d t d \tau\right.}}{\left(1+t \frac{P_{i}}{S_{i}} \epsilon_{i}^{2} x_{i}^{-\alpha}\right)\left(1+\tau \frac{P_{i}}{S_{i}} \epsilon_{i}^{2} x_{i}^{-\alpha}\right)}
\end{gathered}
$$

It is also straightforward to show that

$$
\begin{aligned}
& \mathbb{E} e^{-\frac{P_{i}}{S_{i} x_{i}^{\alpha}}\left(t \hat{H}_{x_{i}, l_{i}}^{\mathrm{MRC}}+\tau \hat{H}_{x_{i}, l_{i}^{\prime}}^{\mathrm{MRRC}}\right.}=\mathbb{E} e^{-\frac{P_{i}}{S_{i} x_{i}^{\alpha}}\left(t \sum_{l^{\prime} \neq l_{i}} \frac{\left\|\tilde{h}_{x_{i}, l_{i}}^{\dagger} \boldsymbol{h}_{x_{i}, l^{\prime}}\right\|^{2}}{\| \tau} \sum_{l_{x_{i}, l_{i} \|^{2}}} \frac{\left\|\tilde{h}_{x_{i}, l_{i}^{\prime}}^{\dagger} \boldsymbol{h}_{x_{i}, l^{\prime \prime}}\right\|^{2}}{\| l_{i}^{\prime}}\right)}
\end{aligned}
$$

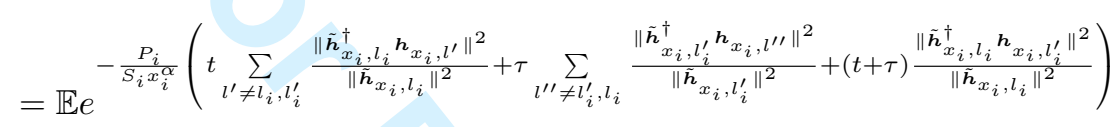

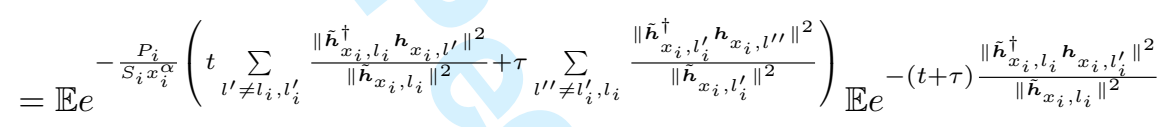

$$
\begin{aligned}
& =\frac{1}{\left(\left(1+t \frac{P_{i}}{S_{i}} x_{i}^{-\alpha}\right)\left(1+\tau \frac{P_{i}}{S_{i}} x_{i}^{-\alpha}\right)\right)^{S_{i}-2}} \frac{1}{1+(t+\tau) \frac{P_{i}}{S_{i}} x_{i}^{-\alpha}} .
\end{aligned}
$$

Inserting (30) in (29), yields:

$$
\begin{aligned}
& \mathcal{I}=\int_{0}^{\infty} \int_{0}^{\infty} \frac{\left(1+(t+\tau) \frac{P_{i}}{S_{i}} x_{i}^{-\alpha}\right)^{-1} \prod_{j \in \mathcal{K}} \mathbb{E}_{\Phi_{j}} \prod_{x_{j} \in \Phi_{j} / x_{i}} \mathbb{E}_{G_{x_{j}, l_{i}}^{\mathrm{MRC}}, G_{x_{j}, l_{i}^{\prime}}^{\mathrm{MRC}}} e^{-\frac{P_{j}\left(t G_{x_{j}, l_{i}^{\prime}}^{\mathrm{MRC}}+G_{\left.x_{j}, l_{i}^{\prime}\right)}^{\mathrm{MRC}}\right.}{S_{j}\left\|x_{j}\right\| \|^{\alpha}}}}{\left(1+t \frac{P_{i}}{S_{i}} \epsilon_{i}^{2} x_{i}^{-\alpha}\right)\left(1+\tau \frac{P_{i}}{S_{i}} \epsilon_{i}^{2} x_{i}^{-\alpha}\right)\left(\left(1+t \frac{P_{i}}{S_{i}} x_{i}^{-\alpha}\right)\left(1+\tau \frac{P_{i}}{S_{i}} x_{i}^{-\alpha}\right)\right)^{S_{i}-2}} d t d \tau \\
& =\int_{0}^{\infty} \int_{0}^{\infty} \frac{\left(1+(t+\tau) \frac{P_{i}}{S_{i}} x_{i}^{-\alpha}\right)^{-1} e^{-\tilde{C}(\alpha) \sum_{j} \lambda_{j}\left(\frac{P_{j}}{S_{j}}\right)^{\check{\alpha}} \bar{W}_{j}(t, \tau)}}{\left(1+t \frac{P_{i}}{S_{i}} \epsilon_{i}^{2} x_{i}^{-\alpha}\right)\left(1+\tau \frac{P_{i}}{S_{i}} \epsilon_{i}^{2} x_{i}^{-\alpha}\right)\left(\left(1+t \frac{P_{i}}{S_{i}} x_{i}^{-\alpha}\right)\left(1+\tau \frac{P_{i}}{S_{i}} x_{i}^{-\alpha}\right)\right)^{S_{i}-2}} d t d \tau,
\end{aligned}
$$

where in the last step we apply (25) in which $\bar{W}_{j}(t, \tau)$ is $\bar{W}_{j}(t, \tau)=\mathbb{E}\left[\left(t G_{x_{j}, l_{i}^{\prime}}^{\mathrm{MRC}}+\tau G_{x_{j}, l_{i}^{\prime}}^{\mathrm{MRC}}\right)^{\check{\alpha}}\right]$.

This is then simplified to (13) noting the independence of $G_{x_{j}, l_{i}^{\prime}}^{\mathrm{MRC}}$, and $G_{x_{j}, l_{i}^{\prime}}^{\mathrm{MRC}}$. The proof is completed by obtaining (12) through combining (28) and (31), and inserting the result as well as the obtained formulas for $\operatorname{Var}\left(\mathrm{SIR}_{x_{i}, l_{i}}^{\mathrm{svd}}\right)$ and $\overline{\mathrm{SIR}}_{x_{i}, l_{i}}^{\mathrm{MRC}}$ into the definition of SIR correlation coefficient in (11). 


\section{REFERENCES}

[1] M. G. Khoshkholgh et al., "Coverage performance of MIMO-MRC in heterogeneous networks: a stochastic geometry perspective," in Proc. of the IEEE VTC-Fall, 2016, [online] http://eprints.lancs.ac.uk/79820/1/K-tier-MIMO-MRC-VTC.pdf.

[2] J. G. Andrews et al., "What will 5G be?” IEEE JSAC, vol. 32, no. 6, pp. 1065-1082, Jun. 2014.

[3] F. Boccardi et al., "Five disruptive technology directions for 5G," IEEE Comm. Mag., vol. 54, no. 2, pp. 74-80, Feb. 2014.

[4] D. Tse and P. Viswanath, Fundamentals of Wireless Communication. Cambridge University Press, September 2004.

[5] A. Lozano et al., "Fundamental limits of cooperation,” IEEE Trans. Inf. Theory, vol. 59, no. 9, pp. 5213-5226, Sep. 2013.

[6] H. S. Dhillon et al., "Modeling and analysis of $k$-tier downlink heterogeneous cellular network," IEEE JSAC, vol. 30, no. 3, pp. 550-560, Apr. 2012.

[7] H. S. Jo et al., "Heterogeneous cellular networks with flexible cell association: A compehensive downlink SINR analysis," IEEE Trans. Wireless Comm., vol. 11, no. 10, pp. 3484-3495, Oct. 2012.

[8] A. Guo and M. Haenggi, "Spatial stochastic models and metrics for the structure of base stations in cellular networks," IEEE Trans. Wireless Comm., vol. 12, no. 11, pp. 5800-5812, Nov. 2013.

[9] J. G. Andrews et al., "A tractable approach to coverage and rate in cellular networks," IEEE Trans. on Comm., vol. 59, no. 11, pp. 3122-3134, Nov. 2011.

[10] M. D. Renzo et al., "The intensity matching approach: A tractable stochastic geometry approximation to system-level analysis of cellular networks," IEEE Trans. Wireless Comm., vol. 15, no. 9, pp. 5963-5983, Sep. 2016.

[11] M. D. Renzo and P. Guan, "Stochastic geometry modeling of coverage and rate of cellular networks using the Gil-Pelaez inversion theorem," IEEE Commun. Lett., vol. 18, no. 9, pp. 1575-1578, Sep. 2014.

[12] C. Li et al., "Analysis of area spectral efficiency and link reliability in multiuser MIMO HetNets," in Proc. IEEE Int. Conf. Commun. (ICC), Jun. 2015.

[13] A. K. Gupta et al., "Downlink multi-antenna heterogeneous cellular network with load balancing," IEEE Trans. Comm., vol. 62, no. 11, pp. 4052-4067, Nov. 2014.

[14] S. T. Veetil, "Performance of PZF and MMSE receivers in cellular networks with multi-user spatial multiplexing," IEEE Trans. Wireless Comm., vol. 14, no. 9, pp. 4867-34 878, Sep. 2015.

[15] Z. Chen et al., "Area spectral efficiency analysis and energy consumption minimization in multi-antenna poisson distributed networks," IEEE Trans. Wireless. Comm., vol. 15, no. 7, pp. 4862-4874, Jul. 2016.

[16] H. H. Yang et al., "Energy-efficient design of MIMO heterogeneous networks with wireless backhaul," IEEE Trans. Wireless. Comm., vol. 15, no. 7, pp. 4914-4927, Jul. 2016.

[17] R. Hernandez-Aquino et al., "Energy efficiency analysis of two-tier MIMO diversity schemes in poisson cellular networks," IEEE Trans. Comm., vol. 63, no. 10, pp. 3898-3911, Oct. 2015.

[18] Y. Wu et al., "Analysis and optimization of inter-tier interference coordination in downlink multi-antenna HetNets with offloading," IEEE Trans. Wireless. Comm., vol. 14, no. 12, pp. 6550-6564, Dec. 2015.

[19] A. Shojaeifard et al., "Design, modeling, and performance analysis of multi-antenna heterogeneous cellular networks," IEEE Trans. Comm., vol. 64, no. 7, pp. 3104-3118, Jul. 2016. 
[20] T.-X. Zheng et al., "Multi-antenna transmission in downlink heterogeneous cellular networks under a threshold-based mobile association policy," IEEE Trans. Comm., 2016.

[21] R. Tanbourgi et al., "Analysis of joint transmit-receive diversity in downlink mimo heterogeneous cellular networks," IEEE Trans. Wireless. Comm., vol. 14, no. 12, pp. 6695-6709, Jul. 2015.

[22] M. D. Renzo and P. Guan, "Stochastic geometry modeling, system-level analysis and optimization of uplink heterogeneous cellular networks with multi-antenna base stations," IEEE Trans. Comm., vol. 64, no. 6, pp. 2453-2476, Jun. 2016.

[23] — "A mathematical framework to the computation of the error probability of downlink MIMO cellular networks by using stochastic geometry," IEEE Trans. Comm., vol. 62, no. 8, pp. 2860-2879, Aug. 2014.

[24] M. D. Renzo and W. Lu, "Stochastic geometry modeling and performance evaluation of MIMO cellular networks using the equivalentin- distribution (EiD)-based approach," IEEE Trans. Comm., vol. 63, no. 3, pp. 977-996, Mar. 2015.

[25] L. H. Afify et al., "Unified stochastic geometry model for MIMO cellular networks with retransmissions," CoRR, vol. arXiv:1604.02960, 2016, [Online]. http://arxiv.org/abs/1604.02960.

[26] S. Mukherjee, "Distribution on downlink SINR in heterogeneous cellular network," IEEE JSAC, vol. 30, no. 3, pp. 575-585, Apr. 2012.

[27] P. Madhusudhanan et al., "Analysis of downlink connectivity models in aheterogenous cellular network via stochastic geometry," IEEE Trans. Wireless. Comm., vol. 15, no. 6, pp. 3895-3907, Jun. 2016.

[28] H. S. Dhillon et al., "Downlink MIMO HetNets: Modeling, ordering results and performance analysis," IEEE Trans. Wireless. Comm., vol. 12, no. 10, pp. 5208-5222, Oct. 2012.

[29] M. G. Khoshkholgh et al., "Performance evaluation of MISO-SDMA in heterogenous networks with practical cell association," in Proc. IEEE VTC-Fall, 2016, [online] http://eprints.lancs.ac.uk/79817/1/K-tier-MIMO-SDMA-HCN-VTC.pdf.

[30] K. Huang et al., "Spatial interference cancellation for multiantenna mobile ad hoc networks," IEEE Trans. Inf. Theory, vol. 58, no. 3, pp. 1660-1676, March 2012.

[31] R. Vaze and R. W. H. Jr., "Transmission capacity of ad-hoc networks with multiple antennas using transmit stream adaptation and interference cancellation," IEEE Trans. Inf. Theory, vol. 58, no. 2, pp. 780-792, Feb. 2012.

[32] R. H. Y. Louie et al., "Open-loop spatial multiplexing and diversity communications in ad hoc networks," IEEE Trans. Inf. Theory, vol. 57, no. 1, pp. 317-344, Jan. 2011.

[33] Y. Wu et al., "Generalized framework for the analysis of linear MIMO transmission schemes in decentralized wireless Ad Hoc networks," IEEE Trans. Wireless. Comm., vol. 11, no. 8, pp. 2815-2827, Aug. 2012.

[34] M. G. Khoshkholgh et al., "Coverage performance in multi-stream MIMO-ZFBF heterogeneous networks," to appear in IEEE Trans. on Vehicular Technology, 2016, [online] http://eprints.lancs.ac.uk/83037/1/khosh.pdf.

[35] M. Haenggi, "Diversity loss due to interference correlation," IEEE Comm. Lett., vol. 16, no. 10, pp. 1600-1603, Oct. 2012.

[36] R. Tanbourgi et al., "Dual-branch MRC receivers in the cellular downlink under spatial interference correlation," in Proc. European Wireless, pp. 13-18, 2014, berlin, Offenbach, Germany.

[37] V. Chandrasekhar et al., "Coverage in multi-antenna two-tier networks," IEEE Trans. Wireless. Comm., vol. 8, no. 10, pp. 5314-5327, Oct. 2009.

[38] J. F. C. Kingman, Poisson Processes. Oxford University Press, 1993. 
[39] M. Haenggi and R. K. Ganti, "Interference in large wireless networks," Foundations and Trends in Networking, vol. 3, no. 2, 2008, Available at http://www.nd.edu/ mhaenggi/pubs/now.pdf.

[40] K. S. Ahn, "Performance analysis of MIMO-MRC systems with channel estimation error in the presence of cochannel interferences," IEEE Sig. Proc. Let., vol. 15, pp. 445-448, 2008.

[41] Y. Chen and C. Tellambura, "Performance analysis of maximum ratio transmission with imperfect channel estimation," IEEE Comm. Let., vol. 9, no. 4, pp. 322-324, Apr. 2005.

[42] J. R. W. Heath and D. J. Love, "Multimode antenna selection for spatial multiplexing systems with linear receivers," IEEE Trans. Signal. Proc., vol. 53, no. 8, pp. 3042-3056, Aug. 2005.

[43] C. B. Papadias and G. J. Foschini, "On the capacity of certain spacetime coding schemes," EURASIP J. Appl. Signal. Proc., vol. 5, pp. 447-458, May 2002.

[44] S. Jin et al., "MIMO multichannel beamforming: SER and outage using new eigenvalue distributions of complex noncentral wishart matrices," IEEE Trans. Comm., vol. 56, no. 3, pp. 424-434, March 2008.

[45] K. R. Kumar et al., "Asymptotic performance of linear receivers in MIMO fading channels," IEEE Trans. Inf. Theory, vol. 55, no. 10, pp. 4398-4418, Oct. 2009.

[46] L. G. Ordonez et al., "Ordered eigenvalues of a general class of hermitian random matrices with application to the performance analysis of MIMO systems," IEEE Trans. Signal. Proc., vol. 57, no. 2, pp. 672-689, Feb. 2006.

[47] A. M. Hunter and J. G. Andrews, "Adaptive rate control over multiple spatial channels in ad hoc networks," in 6th International Symposium of IEEE WiOPT, pp. 469-474, Apr. 2008.

[48] R. Tanbourgi et al., "Analysis of joint transmitreceive diversity in downlink MIMO heterogeneous cellular networks," IEEE Trans. Wireless Comm., vol. 14, no. 12, pp. 6695-6709, Dec. 2015.

[49] M. G. Khoshkholgh and V. C. M. Leung, "On the performance of MIMO-SVD multiplexing systems in hetnets: A stochastic geometry perspective," to appear in IEEE Trans. on Vehicular Technology, 2017.

[50] M. Haenggi et al., "Stochastic geometry and random graphs for the analysis and design of wireless networks," IEEE JSAC, vol. 27, no. 7, pp. 1029-1046, Sep. 2009. 


\begin{abstract}
We study the coverage performance of multiantenna (MIMO) communications with maximum ratio combining (MRC) at the receiver in heterogeneous networks (HetNets). Our main interest in on multi-stream communications when BSs do not have access to channel state information. Adopting stochastic geometry we evaluate the network-wise coverage performance of MIMO-MRC assuming maximum signal-tointerference ratio (SIR) cell association rule. Coverage analysis in MIMO-MRC HetNets is challenging due to inter-stream interference and statistical dependencies among streams' SIR values in each communication link. Using the results of stochastic geometry we then investigate this problem and obtain tractable analytical approximations for the coverage performance. We then show that our results are adequately accurate and easily computable. Our analysis sheds light on the impacts of important system parameters on the coverage performance, and provides quantitative insight on the densification in conjunction with high multiplexing gains in MIMO HetNets. We further observe that increasing multiplexing gain in high-power tier can cost a huge coverage reduction unless it is practiced with densification in femto-cell tier.
\end{abstract}

\section{INTRODUCTION}

Traffic demands of cellular networks-heavily driven by the popularity of video streaming and mobile social networkingare rapidly growing. Densification is one of the main approaches operators are advocating to smoothly deal with this unprecedented deluge of traffic [1]. Spectral efficiency is expectedly growing substantially because of small communication distance and universal frequency reuse. Much better performance will be, on the other hand, materialized by vastly exploiting multi-antenna (MIMO) techniques.

Nevertheless, the network performance of MIMO communications in conjunction with densification and heterogeneity is yet to be truly understood. One way to pave the road for such comprehensive assessment of the network can be achieved by adopting tools from stochastic geometry. It has been vastly employed for evaluation of the various performance metrics in wireless networks including heterogeneous networks (HetNets), see, e.g., [2], [3]. Work of [2] has proposed a flexible approach in modeling the network by $K$ tiers of independent Poisson point processes (PPPs) and maximum signalto-interference ratio (SIR) for purpose of cell association (CA). Authors in [4] have then used the framework of [2] for studying the coverage and rate performances of MISO HetNets. By providing ordering results, it has been shown that in some scenarios space-division multiple access (SDMA) was an inferior scheme to single user eigen-beamforming. In [5], [6] area spectral efficiency of MISO-SDMA systems has investigated assuming CA rule of maximum average received power. In [7] the outage performance of space-time block codes at the transmitters and optimal combining received filters have studied. Work of [8] has focused on the advantages of interference cancellation in zero-forcing based received filters in enhancing the coverage performance of cellular systems. However, the CA rules of [7], [8], [9] are simply a duplicate of the counterpart in single-antenna (SISO) systems of e.g., [3]. It is then very compelling to develop analysis based on CA rules that comprehensively encompass the traits of MIMO communications in improving multiplexing and diversity. For this reason, we here focus on maximum SIR rule.

We chiefly focus on multi-stream MIMO-MRC HetNets. Despite the practical significance of MIMO-MRC - chiefly because of its straightforward implementations, affordable computational complexities, and near to zero feedback overheads - the literature dealing with its performance in HetNets is small. This is because of inherent complexities rooting from residual interference among data streams each communication link suffers from. It was however previously studied in the literature of ad hoc communications, see. e.g., [10]. But, in comparison to cellular systems in ad hoc communications the network configuration lacks CA stage, which render inapplicability of the derived results therein for HetNets. Besides, in the literature of MIMO communications, both ad hoc and cellular systems, the coverage probability per a data stream was merely studied, while in reality coverage probability per a communication link (global coverage probability) comprising of multiple streams is the main performance metric. To bridge such gaps, we therefore provide accurate approximations on the latter metric via analysis. The derived bounds explicitly capture the impacts of important system parameters such as density of BSs and multiplexing gains. Our results, further, indicate that, in general, increasing multiplexing gains worsens the coverage performance of HetNets.

\section{SYSTEM MODEL}

Consider downlink communication paradigms in heterogeneous cellular networks (HetNets) comprising of $K \geq 1$ tiers of randomly located BSs. BSs of tier $i \in \mathcal{K}$ are spatially 
distributed according to a homogenous Poisson Point Process (PPP) $\Phi_{i}$ with given spatial density $\lambda_{i} \geq 1$ [2]. For mathematical tractability we assume that the processes are mutually independent. Each tier $i$ can entirely be characterized with the parameters: spatial density of BSs $\lambda_{i}$, transmission power of BSs $P_{i}$ Watts, SIR threshold $\beta_{i} \geq 1$, number of BS's transmit antennas $N_{i}^{t}$, and finally the number of scheduled streams $S_{i} \leq \min \left\{N_{i}^{t}, N^{r}\right\} . S_{i}$ is referred to as multiplexing gain here. Also, $N^{r}$ is the number of antennas user equipments (UEs) possess. The modelled system of multi-stream data communication is considered as $S_{i}$ pipes of information as [11], [10]. UEs also randomly scattered across the network and form a PPP $\Phi_{U}$, independent of $\left\{\Phi_{i}\right\}$ s, with density $\lambda_{U} \gg \sum_{i} \lambda_{i}$. At each given time slot only one UE is served per active cell [12], [4], [7]. In the case that more that one UE is associated with a given BS time-sharing per cell is adopted for scheduling.

Note that according to Slivnayak's theorem and thanks to the stationarity of the point processes [13], [14], the spatial performance of the network can be adequately obtained from the eye of a typical $U E$ positioned at the origin. Let the typical UE be associated with BS $x_{i}$ transmitting $S_{i}$ data streams. Denote $\boldsymbol{y}_{x_{i}} \in \mathbb{C}^{N^{r} \times 1}$ as the received signal:

$$
\boldsymbol{y}_{x_{i}}=\left\|x_{i}\right\|^{-\frac{\alpha}{2}} \boldsymbol{H}_{x_{i}} \boldsymbol{s}_{x_{i}}+\sum_{j \in \mathcal{K}} \sum_{x_{j} \in \Phi_{j} / x_{0}}\left\|x_{i}\right\|^{-\frac{\alpha}{2}} \boldsymbol{H}_{x_{j}} \boldsymbol{s}_{x_{j}},
$$

where $s_{x_{i}}=\left[s_{x_{i}, 1} \ldots s_{x_{i}, S_{i}}\right]^{T} \in \mathbb{C}^{S_{i} \times 1}$, so that $s_{x_{i}, l} \sim$ $\mathcal{C N}\left(0, P_{i} / S_{i}\right)$, is the transmitted streams at BS $x_{i}, \boldsymbol{H}_{x_{i}} \in$ $\mathbb{C}^{N^{r} \times S_{i}}$ is the intended fading channel matrix between BS $x_{i}$ and the typical UE with entries independently drawn from $\mathcal{C} \mathcal{N}(0,1)$, i.e., Rayleigh fading assumption. Transmitted signals are assumed independent. Likewise, channel matrices are independent. $\left\|x_{i}\right\|^{-\alpha}$ is the distance-dependent path-loss attenuation where $\left\|x_{i}\right\|$ denotes the Euclidian distance between BS $x_{i}$ and the origin, and $\alpha>2$ is the path-loss exponent. We further define $\check{\alpha}=2 / \alpha$. We assume that the typical UE knows $\boldsymbol{H}_{x_{i}}$ perfectly-perfect CSI at the receiver (CSIR).

We focus on the scenarios that BSs do not have access to the channel state information at the transmitter (CSIT). Thus BSs of each tier $i$ simply turn on $S_{i}$ transmit antennas and air information-bearing signals with fixed transmission power $P_{i}$ that is equally divided among the transmitted data streamsopen-loop technique [10], [11]. For the specific purpose of this paper maximum ratio combining (MRC) at the receiver is considered. Accordingly, for decoding $l_{i}$-th stream of data the typical receiver extracts $l_{i}$-th column of matrix $\boldsymbol{H}_{x_{i}}$ and multiplies its corresponding conjugate with the received vector (1). Let r.v.s $H_{x_{i}, l_{i}}^{\mathrm{mrc}}$ be chi-squared with $2 N^{r} \mathrm{DoFs}, \tilde{H}_{x_{i}, l_{i}}^{\mathrm{mrc}}$ be chi-squared with $2\left(S_{i}-1\right)$ DoFs, and $G_{x_{j}, l_{i}}^{\mathrm{mrc}}$ be chi-squared with DoF $2 S_{j}$, respectively, standing for the intending channel power gains associated with $l_{i}$-th data stream, inter-stream interference on stream $l_{i}$ caused by streams $l_{i}^{\prime} \neq l_{i}$, and inter-cell interference (ICI) caused by BSs $x_{j} \neq x_{i}$ on data stream $l_{i}$. Regarding [10] we can show that the SIR expression associated with $l_{i}$-th data stream is

$$
\operatorname{SIR}_{x_{i}, l_{i}}^{\mathrm{mrc}}=\frac{\frac{P_{i}}{S_{i}}\left\|x_{i}\right\|^{-\alpha} H_{x_{i}, l_{i}}^{\mathrm{mrc}}}{\frac{P_{i}}{S_{i}}\left\|x_{i}\right\|^{-\alpha} \tilde{H}_{x_{i}, l_{i}}^{\mathrm{mrc}}+\sum_{j \in \mathcal{K}} \sum_{x_{j} \in \Phi_{j} / x_{i}} \frac{P_{j}}{S_{j}}\left\|x_{j}\right\|^{-\alpha} G_{x_{j}, l_{i}}^{\mathrm{mrc}}} .
$$

Per each stream and across streams all fading coefficients are independent. Also, (2) is identically, but not independently, distributed across streams. The nominator and denominator of (2) are respectively represent the effective power of intended signal of stream $l_{i}$ and inter-stream interference plus ICI.

\section{Coverage Analysis}

We merely consider fixed-rate transmission (FRT) scheme, in which the transmission rate on each stream $l_{i}$ is constant, and equal to $R_{x_{i}, l_{i}}=\log \left(1+\beta_{i}\right) \mathrm{bit} / \mathrm{sec} / \mathrm{Hz}$, assuming that the typical UE is associated with $\mathrm{BS} x_{i}$. Typical UE is associated with the best BS that its weakest stream is stronger than the corresponding SIR threshold. To declare the coverage per communication link, FRT scheme mandates that at all $S_{i}$ scheduled streams the corresponding SIR values satisfy the required SIR threshold $\beta_{i} \geq 1$, i.e., the typical UE is claimed to be in coverage if set

$$
\mathcal{A}_{\text {frt }}=\left\{\exists i \in \mathcal{K}: \max _{x_{i} \in \Phi_{i} l_{i}=1, \ldots, S_{i}} \min _{x_{i}, l_{i}}^{\operatorname{mrc}} \geq \beta_{i}\right\},
$$

is nonempty. We therefore define coverage probability $o_{\mathrm{frt}}^{\mathrm{mrc}}=$ $\mathbb{P}\left\{\mathcal{A}_{\text {frt }} \neq \emptyset\right\}$. Note that exact evaluation of $o_{\mathrm{frt}}^{\mathrm{mrc}}$ is very complex mainly because of dependency of SIR values (2) across streams per each communication link as well as the inter-stream interference on each stream. We thus in the following resort to approximating the coverage probability.

Proposition 1: With MIMO-MRC and maximum SIR CA rule, the coverage performance can be approximated as

$o_{\mathrm{frt}}^{\operatorname{mrc}} \leq \sum_{i \in \mathcal{K}} \frac{\frac{\pi}{\tilde{C}(\alpha)}\left(\frac{P_{i}}{S_{i} \beta_{i}}\right)^{\check{\alpha}} \frac{\lambda_{i}}{S_{i}^{\check{\alpha}}}\left(\left.\sum_{r_{i}=0}^{N^{r}-1} \frac{(-1)^{r_{i}}}{r_{i} !} \frac{d^{r_{i}}}{d t^{r_{i}}} \frac{t^{-\frac{\check{\alpha}}{S_{i}}}}{\left(1+t \beta_{i}\right)^{S_{i}-1}}\right|_{t=1}\right)^{S_{i}}}{\sum_{j=1}^{K} \lambda_{j}\left(\frac{P_{j}}{S_{j}}\right)^{\check{\alpha}}\left(\frac{\Gamma\left(\frac{\check{\alpha}}{S_{i}}+S_{j}\right)}{\Gamma\left(S_{j}\right)}\right)^{S_{i}}}$.

Proof: See the Appendix.

(4) demonstrates impacts of many important system parameters such as deployment density, transmission power, multiplexing gain, and SIR threshold of tiers. Note that, in general the nominator and denominator of (4) are respectively corresponding to the intended communication link and ICI. On the other hand, the impact of inter-stream interference is captured by $\left(1+t \beta_{i}\right)^{S_{i}-1}$ that solely depends on SIR threshold and multiplexing gain.

Please note that evaluation of (4) is actually computationally affordable. But, it is yet possible to provide bounds excluding the evaluation of high-order derivatives as is carried out in following:

Proposition 2: Another approximation on the coverage probability of MIMO-MRC system with maximum SIR CA 
rule might be

$$
o_{\mathrm{frt}}^{\operatorname{mrc}} \approx \frac{\pi}{\tilde{C}(\alpha)} \sum_{i \in \mathcal{K}} \frac{\lambda_{i}\left(\frac{P_{i}}{S_{i}^{2} \beta_{i}}\right)^{\check{\alpha}}\left(\frac{\sum_{r_{i}=0}^{N^{r}-1} \frac{\Gamma\left(\frac{\check{\alpha}}{S_{i}}+\frac{S_{i}-1}{2}+r_{i}\right)}{\Gamma\left(\frac{\check{\alpha}}{S_{i}}+\frac{S_{i}-1}{2}\right) \Gamma\left(1+r_{i}\right)}}{\sum_{l_{i}=0}^{S_{i}-1}\left(\begin{array}{c}
S_{i}-1 \\
l_{i}
\end{array}\right) \beta_{i}^{l_{i}}}\right)^{S_{i}}}{\sum_{j \in \mathcal{K}} \lambda_{j}\left(\frac{P_{j}}{S_{j}}\right)^{\check{\alpha}}\left(\frac{\Gamma\left(\frac{\check{\alpha}}{S_{i}}+S_{j}\right)}{\Gamma\left(S_{j}\right)}\right)^{S_{i}}},
$$

Proof: To prove this claim, we apply the following heuristic approximation

$\left(1+t_{i} \beta_{i}\right)^{S_{i}-1}=\sum_{l_{i}=0}^{S_{i}-1}\left(\begin{array}{c}S_{i}-1 \\ l_{i}\end{array}\right) \beta_{i}^{l_{i}} t_{i}^{l_{i}} \approx t_{i}^{\frac{S_{i}-1}{2}} \sum_{l_{i}=0}^{S_{i}-1}\left(\begin{array}{c}S_{i}-1 \\ l_{i}\end{array}\right) \beta_{i}^{l_{i}}$.

Using this, equation (10) in appendix is reduced to

$=\sum_{i \in \mathcal{K}} \frac{\frac{\pi}{\tilde{C}(\alpha)}\left(\frac{P_{i}}{S_{i} \beta_{i}}\right)^{\check{\alpha}} \frac{\lambda_{i}}{S_{i}^{\check{\alpha}}}\left(\begin{array}{c}S_{i}-1 \\ l_{i}=0\end{array}\left(\begin{array}{c}S_{i}-1 \\ l_{i}\end{array}\right) \beta_{i}^{l_{i}}\right)^{-S_{i}}}{\sum_{j=1}^{K} \lambda_{j}\left(\frac{P_{j}}{S_{j}}\right)^{\check{\alpha}}\left(\frac{\Gamma\left(\frac{\check{\alpha}}{S_{i}}+S_{j}\right)}{\Gamma\left(S_{j}\right)}\right)^{S_{i}}}\left(\int_{0}^{\infty} \frac{\mathcal{L}_{\overline{\mathrm{F}}_{H_{i}^{\mathrm{mrc}}}}\left(t_{i}\right)}{t_{i}^{\frac{\check{S}}{S_{i}}} \frac{S_{i}-1}{2}}\right)^{S_{i}}$.

The claimed result is then obtained recalling that $H_{i}^{\mathrm{rmc}}$ is chisquared r.v. with DoF $2 N^{r}$.

Corollary 1: Let $S_{i}=1 \forall i$ and $N^{r}=1$, thus (4) is reduced to $o_{\text {SISO }}=\frac{\pi}{C(\alpha)} \frac{\sum_{i \in \mathcal{K}} \lambda_{i}\left(\frac{P_{i}}{\beta_{i}}\right)^{\check{\alpha}}}{\sum_{j \in \mathcal{K}} \lambda_{j} P_{j}^{\check{\alpha}}}$, which coincides with the result of [2] of single-antenna (SISO) HetNets.

Corollary 2: Let $S_{i}=1 \forall i$ thus o $o_{\mathrm{SIMO}}=$ $O_{\mathrm{SISO}} \sum_{r=0}^{N^{r}-1} \frac{\Gamma(\check{\alpha}+r)}{\Gamma(\check{\alpha}) \Gamma(1+r)}$.

Note that in the case of SIMO scenario the results are actually accurate. On the other hand, by comparing SISO and SIMO cases it is easy to confirm that

$$
\frac{o_{\text {SIMO }}}{o_{\text {SISO }}}=\sum_{r=0}^{N^{r}-1} \frac{\Gamma(\check{\alpha}+r)}{\Gamma(\check{\alpha}) \Gamma(1+r)} .
$$

Using this result, one may show that by applying Kershaws inequality [15] we have

$\Gamma(\check{\alpha}) \frac{o_{\text {SIMO }}}{o_{\text {SISO }}} \geq \sum_{r=0}^{N^{r}-1}(r+0.5 \check{\alpha})^{\check{\alpha}-1} \approx \int_{0}^{N^{r}-1}(z+0.5 \check{\alpha})^{\check{\alpha}-1} d z$

or equivalently $\frac{o_{\mathrm{SIMO}}^{\mathrm{zf}}}{o_{\mathrm{SISO}}} \propto\left(N^{r}\right)^{\check{\alpha}}$.

\section{Simulation Results}

In this section we present simulation results. For clarity we set $K=2$. The simulation results are based on Monte Carlo technique.

We study the accuracy of the analytical findings of the paper against deploying densities of the BSs in Fig. 1 and Fig. 2. In the former (the later) we fix $\lambda_{1}=10^{-4}\left(\lambda_{2}=10^{-4}\right)$ and change $\lambda_{2}\left(\lambda_{1}\right)$. As it is seen Proposition 1 provides accurate bound on the coverage probability while the accuracy of Proposition 2 is generally questionable. However, there are scenarios, see, Fig. 2 case of $S_{1}=6$ and $S_{2}=2$, that Proposition 2 is also accurate.
Fig. 1. $\lambda_{1}=10^{-4} \cdot \alpha=4, N^{r}=10, P_{1}=50 \mathrm{~W}, P_{1}=10 \mathrm{~W}, \beta_{1}=2$, and $\beta_{2}=5$.
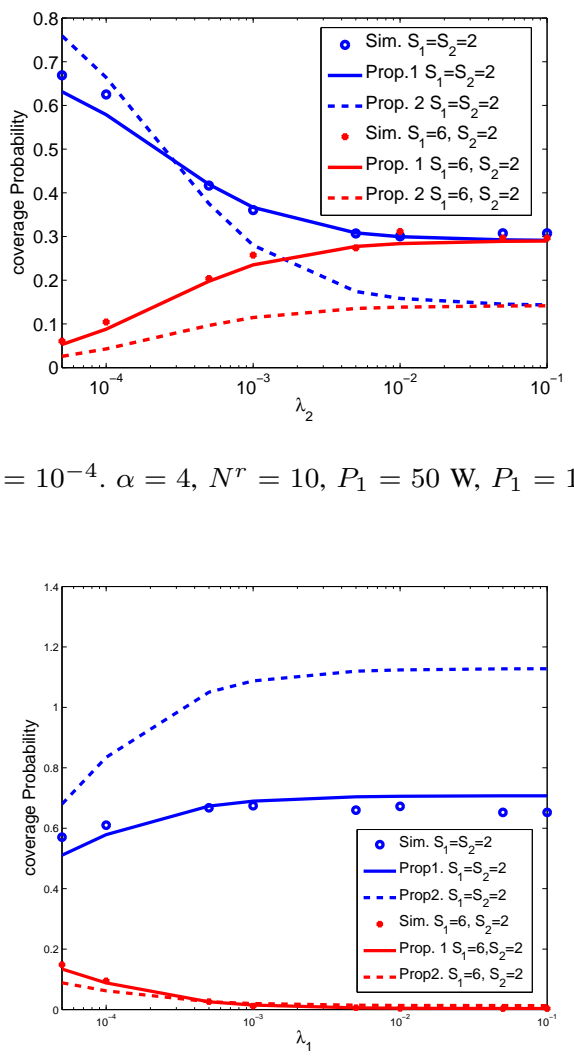

Fig. 2. $\lambda_{2}=10^{-4} . \alpha=4, N^{r}=10, P_{1}=50 \mathrm{~W}, P_{1}=10 \mathrm{~W}, \beta_{1}=2$, and $\beta_{2}=5$.

Moreover, both of these illustrations highlight many important trends showing the impacts of multiplexing gains and densifications on the coverage performance.

First, as Fig. 1 reveals when $\lambda_{1}$ is fixed (the density of high-power BSs in tier 1) by increasing $\lambda_{2}$ smaller coverage will follow if $S_{1}=S_{2}$. In the contrary, Fig. 2 indicates that when $\lambda_{2}$ is kept fixed (density of low-power BSs in tier 2) by increasing $\lambda_{1}$ higher coverage performance is resulted again when $S_{1}=S_{2}$. In fact, for the cases that the multiplexing gains are the same across the tiers, the coverage probability could decrease/increase depends upon the tier that the densification is practiced in. The findings of these illustrations indicate that for such cases it is better to densify the tier with higher transmission power.

Second, on the other hand, Fig. 1 shows that for fixed $\lambda_{1}$, increasing $\lambda_{2}$ is beneficial and renders higher coverage performance when $S_{1}=6$ and $S_{2}=2$. Fig. 2 further highlights that when $S_{1}=6$ and $S_{2}=2$ and $\lambda_{2}$ is fixed, increasing $\lambda_{1}$ extremely exacerbates the coverage probability. Consequently, in scenarios that multiplexing gains are not the same it is better to densify the tier corresponding to low-power and low multiplexing gain.

Third, for high values of $\lambda_{2}$ Fig. 1 indicates that both scenarios of $S_{1}=6, S_{2}=2$ and $S_{1}=S_{2}=2$ perform the same. While, Fig. 2 indicates that for high values of $\lambda_{1}$ there is a huge gap between the coverage probability of regime $S_{1}=6$, 
$S_{2}=2$ and coverage probability of regime $S_{1}=S_{2}=2$. In the other words, when the network is ultra-dense in low-power tier, it is possible to increase the multiplexing gain of highpower tier without worrying about the coverage performance.

In summary, the above observations suggest that increasing the density of low power BSs (tier two) should be interpreted as a welcome for the growth of the multiplexing gains of tier 1 without damaging the coverage performance. Moreover, if we are allowed to practice densification in tier 1 , it could render higher performance provided that the similar multiplexing gains are set across the all tiers.

According to the results of both Fig. 1 and Fig. 2 we observe that increasing the density of low power BSs of tier 2 yields a much profound impact on the coverage probability than does tier 1 . For example, 10 fold densification of tier 2 (tier 1) changes the coverage performance by more than $300 \%(100 \%)$. This is actually very important from practical viewpoints because installing more low-power BSs is more economically feasible than increasing the density of highpower BSs of tier 1. Finally, both of these figures confirm that for large values of $\lambda_{1}$ as well as $\lambda_{2}$ the coverage probability is stable and does not responde to densities, which is known as scale invariancy phenomenon in the literature [2].

\section{CONCLUSIONS}

We studied the coverage performance of multi-antenna (MIMO) communications with multi-stream maximum ratio combining (MRC) at the receiver in heterogenous networks (HetNets) when BSs did not have access to channel state information. We utilized powerful tools of stochastic geometry and PPP to comprehensively evaluate the network-wise coverage performance of MIMO-MRC when the cell association rule was maximum signal-to-interference ratio (SIR). Our analysis provided accurate, and easy-to-use bound of the coverage performance. Combined with simulations, it further demonstrated various important aspects of denseness and high multiplexing gains in HetNets. It was observed that increasing multiplexing gains could severely damage the coverage probability unless it practiced in high-power, low density tier in conjunction with densified low-power tier.

\section{ACKNOWLEDGMENT}

This work was supported in part by a UBC Four Year Doctoral Fellowship, by the Canadian Natural Sciences and Engineering Research (grants RGPIN-2014-06119 and RGPAS462031-2014), and by the National Natural Science Foundation of China (Grant 61271182).

\section{REFERENCES}

[1] J. G. Andrews et al., "What will 5G be?" IEEE JSAC, vol. 32, no. 6, pp. 1065-1082, Jun. 2014.

[2] H. S. Dhillon et al., "Modeling and analysis of $k$-tier downlink heterogeneous cellular network," IEEE JSAC, vol. 30, no. 3, pp. 550-560, Apr. 2012.

[3] H. S. Jo et al., "Heterogeneous cellular networks with flexible cell association: A compehensive downlink SINR analysis," IEEE Trans. Wireless Comm., vol. 11, no. 10, pp. 3484-3495, Oct. 2012.
[4] H. S. Dhillon et al., "Downlink MIMO hetnets: Modeling, ordering results and performance analysis," IEEE Trans. Wireless. Comm., vol. 12, no. 10 , pp. 5208-5222, Oct. 2012.

[5] C. Li et al., "Success probability and area spectral efficiency in multiuser MIMO HetNets," submitted to IEEE Trans. Comm., 2015.

[6] A. K. Gupta et al., "Downlink multi-antenna heterogeneous cellular network with load balancing," IEEE Trans. Comm., vol. 62, no. 11, pp. 4052-4067, Nov. 2014.

[7] R. Tanbourgi et al., "Analysis of joint transmit-receive diversity in downlink mimo heterogeneous cellular networks," to appear in IEEE Trans. Wireless. Comm., 2015.

[8] S. T. Veetil, "Performance of pzf and mmse receivers in cellular networks with multi-user spatial multiplexing," IEEE Trans. Wireless Comm., vol. 14, no. 9, pp. 4867-34 878, Sep. 2015.

[9] C. Li et al., "Analysis of area spectral efficiency and link reliability in multiuser MIMO HetNets," in Proc. IEEE Int. Conf. Commun. (ICC), Jun. 2015.

[10] R. H. Y. Louie et al., "Open-loop spatial multiplexing and diversity communications in ad hoc networks," IEEE Trans. Inf. Theory, vol. 57, no. 1, pp. 317-344, Jan. 2011.

[11] R. Vaze and R. W. H. Jr., "Transmission capacity of ad-hoc networks with multiple antennas using transmit stream adaptation and interference cancellation," IEEE Trans. Inf. Theory, vol. 58, no. 2, pp. 780-792, Feb. 2012.

[12] V. Chandrasekhar et al., "Coverage in multi-antenna two-tier networks," IEEE Trans. Wireless. Comm., vol. 8, no. 10, pp. 5314-5327, Oct. 2009.

[13] J. F. C. Kingman, Poisson Processes. Oxford University Press, 1993.

[14] M. Haenggi and R. K. Ganti, "Interference in large wireless networks," Foundations and Trends in Networking, vol. 3, no. 2, 2008, Available at http://www.nd.edu/ mhaenggi/pubs/now.pdf.

[15] K. Huang et al., "Spatial interference cancellation for multiantenna mobile ad hoc networks," IEEE Trans. Inf. Theory, vol. 58, no. 3, pp. 1660-1676, Mrc. 2012.

[16] W. C. Ao et al., "Bounds and exact mean node degree and node isolation probability in interference-limited wireless ad hoc networks with general fading," IEEE TVT, vol. 61, no. 5, pp. 2342-2348, Jun. 2012.

[17] M. Haenggi et al., "Stochastic geometry and random graphs for the analysis and design of wireless networks," IEEE JSAC, vol. 27, no. 7, pp. 1029-1046, Sep. 2009.

\section{APPENDIX: Proof of Proposition 1}

According to Lemma 1 in [2] and recalling that we have assumed $\beta_{i} \geq 1 \forall i$, we can write

$$
o_{\mathrm{frt}}^{\mathrm{mrc}}=\sum_{i \in \mathcal{K}} \mathbb{E} \sum_{x_{i} \in \Phi_{i}} 1\left(\min _{l=1, \ldots, S_{i}} \operatorname{SIR}_{x_{i}, l}^{\mathrm{mrc}} \geq \beta_{i}\right) .
$$

(6) is further simplified as:

$$
\begin{aligned}
& o_{\mathrm{frt}}^{\mathrm{mrc}}=\sum_{i \in \mathcal{K}} 2 \pi \lambda_{i} \int_{0}^{\infty} x_{i} \mathbb{P}\left\{\min _{l_{i}=1, \ldots, S_{i}} \mathrm{SIR}_{x_{i}, l_{i}}^{\mathrm{mrc}} \geq \beta_{i}\right\} d x_{i} \\
& =\sum_{i \in \mathcal{K}} 2 \pi \lambda_{i} \int_{0}^{\infty} x_{i} \mathbb{E}_{\left\{\Phi_{j}\right\}} \prod_{l_{i}=1}^{S_{i}} \mathbb{P}\left\{\mathrm{SIR}_{x_{i}, l}^{\mathrm{mrc}} \geq \beta_{i} \mid\left\{\Phi_{j}\right\}\right\} d x_{i}
\end{aligned}
$$

where the first step is because of Campbell's theorem [13], and in step 4 we have used the fact that conditioned on processes $\Phi_{j}$ s SIR values across streams are statistically independent. For fix value of $x_{i}$, we now provide an expression for $\mathbb{P}\left\{\mathrm{SIR}_{x_{i}, l_{i}}^{\mathrm{mrc}} \geq \beta_{i} \mid\left\{\Phi_{j}\right\}\right\}$ as follows

$$
\begin{gathered}
\mathbb{P}\left\{\operatorname{SIR}_{x_{i}, l_{i}}^{\mathrm{mrc}} \geq \beta_{i} \mid\left\{\Phi_{j}\right\}\right\}=\mathbb{P}\left\{H_{x_{i}, l_{i}}^{\mathrm{mrc}} \geq \frac{S_{i} \beta_{i} x_{i}^{\alpha}}{P_{i}} \frac{P_{i}}{S_{i}} x_{i}^{-\alpha} \tilde{H}_{x_{i}, l_{i}}^{\mathrm{mrc}}\right. \\
\left.+\frac{S_{i} \beta_{i} x_{i}^{\alpha}}{P_{i}} \sum_{j \in \mathcal{K}} \sum_{x_{j} \in \Phi_{j} / x_{i}} \frac{P_{j}}{S_{j}}\left\|x_{j}\right\|^{-\alpha} G_{x_{j}, l_{i}}^{\mathrm{mrc}} \mid\left\{\Phi_{j}\right\}\right\} \\
=\int_{0}^{\infty} \mathcal{L}_{\overline{\mathrm{F}}_{H_{i} \mathrm{mrc}}^{-1}}\left(t_{i}\right) \prod_{j \in \mathcal{K}} \prod_{x_{j} \in \Phi_{j} / x_{i}} \mathbb{E}_{G_{x_{j}, l_{i}}^{\mathrm{mrc}} e^{-t_{i} \beta_{i} \frac{S_{i}}{P_{i}} x_{i}^{\alpha} \frac{P_{j}}{S_{j}}\left\|x_{j}\right\|^{-\alpha} G_{x_{j}, l_{i}}^{\mathrm{mrc}}}}
\end{gathered}
$$




$$
\times \mathbb{E} e^{-t_{i} \beta_{i} \tilde{H}_{x_{i}, l_{i}}^{\mathrm{mr}}} d t_{i}
$$

$=\int_{0}^{\infty} \frac{\mathcal{L}_{\overline{\mathrm{F}}_{H_{i}}^{\mathrm{mrc}}}^{-1}\left(t_{i}\right)}{\left(1+t_{i} \beta_{i}\right)^{S_{i}-1}} \prod_{j \in \mathcal{K}} \prod_{x_{j} \in \Phi_{j} / x_{i}} \mathbb{E}_{G_{x_{j}, l_{i}}^{\mathrm{mrc}}} e^{-t_{i} \beta_{i} \frac{S_{i}}{P_{i}} x_{i}^{\alpha} \frac{P_{j}}{S_{j}\left\|x_{j}\right\| \alpha} G_{x_{j}, l_{i}}^{\operatorname{mrc}}} d t_{i}$ where $\mathcal{L}_{\overline{\mathrm{F}}_{H_{i}^{\text {mrc }}}}^{-1}\left(t_{i}\right)$ is the inverse Laplace transform of $H_{i}^{\text {mrc }}$ which is equal to $\mathcal{L}_{\overline{\mathrm{F}}_{H_{i}^{\mathrm{mrc}}}}^{-1}\left(t_{i}\right)=\sum_{m=0}^{N^{r}-1} \frac{1}{m !} \delta^{(m)}(t-1)$ [16], such that $\int_{0}^{\infty} e^{-t_{i} h} \mathcal{L}_{\overline{\mathrm{F}}_{H_{i}^{\mathrm{mrc}}}}^{-1}\left(t_{i}\right) d t_{i}=e^{-h} \sum_{l=0}^{N^{r}-1} \frac{h^{l}}{l !}$, and $\delta^{(m)}(t)$ is the $m$-th derivative of Dirac delta function. Note that in (8) we have discarded index $l_{i}$ from $\mathcal{L}_{\overline{\mathrm{F}}_{H_{i}}^{\text {mrc }}}^{-1}\left(t_{i}\right)$ due to the fact that $H_{x_{i}, l_{i}}^{\mathrm{mrc}}$ are identical r.v.s across streams. Substituting (8) into (7) and applying some straightforward manipulations, it is then seen that

$$
\begin{gathered}
o_{\mathrm{frt}}^{\mathrm{mrc}}=\sum_{i \in \mathcal{K}} 2 \pi \lambda_{i} \int_{0}^{\infty} x_{i} \mathbb{E}_{\left\{\Phi_{j}\right\}} \prod_{l_{i}=1}^{S_{i}} \int_{0}^{\infty} \frac{\mathcal{L}_{\overline{\mathrm{F}}_{H_{i}^{m}}^{\mathrm{mrc}}}^{-1}\left(t_{i}\right)}{\left(1+t_{i} \beta_{i}\right)^{S_{i}-1}} \prod_{j \in \mathcal{K}} \prod_{x_{j} \in \Phi_{j} / x_{i}} \\
\mathbb{E}_{G_{x_{j}, l_{i}}^{\mathrm{mr}}} e^{-t_{i} \beta_{i} \frac{S_{i}}{P_{i}} x_{i}^{\alpha} \frac{P_{j}}{S_{j}}\left\|x_{j}\right\|^{-\alpha} G_{x_{j}, l_{i}}^{\operatorname{mrc}}} d t_{i} d x_{i}
\end{gathered}
$$$$
=\sum_{i \in \mathcal{K}} 2 \pi \lambda_{i} \int_{0}^{\infty} x_{i} d x_{i} \mathbb{E}_{\left\{\Phi_{j}\right\}} \int_{0}^{\infty} \ldots \int_{0}^{\infty} \prod_{j \in \mathcal{K}} \prod_{x_{j} \in \Phi_{j} / x_{i}} \prod_{l_{i}=1}^{S_{i}} \mathbb{E}_{G_{x_{j}, l_{i}}^{\mathrm{mrc}}}
$$$$
e^{-\beta_{i} \frac{S_{i}}{P_{i}} x_{i}^{\alpha} \frac{P_{j}}{S_{j}}\left\|x_{j}\right\|^{-\alpha} G_{x_{j}, l_{i}}^{\operatorname{mrc}} t_{l_{i}}} \prod_{l_{i}=1}^{S_{i}} \frac{\mathcal{L}_{\overline{\mathrm{F}}_{H_{i}^{\mathrm{mrc}}}}^{-1}\left(1+t_{l_{i}}\right)}{\left(1+t_{l_{i}} \beta_{i}\right)^{S_{i}-1}} d t_{l_{i}}
$$$$
=\sum_{i \in \mathcal{K}} 2 \pi \lambda_{i} \int_{0}^{\infty} x_{i} d x_{i} \mathbb{E}_{\left\{\Phi_{j}\right\}} \int_{0}^{\infty} \ldots \int_{0}^{\infty} \prod_{j \in \mathcal{K}} \prod_{x_{j} \in \Phi_{j} / x_{i}} \mathbb{E}_{G_{x_{j}}^{\mathrm{mrc}}}
$$$$
\prod_{l_{i}=1}^{S_{i}} e^{-\beta_{i} \frac{S_{i}}{P_{i}} x_{i}^{\alpha} \frac{P_{j}}{S_{j}}\left\|x_{j}\right\|^{-\alpha} G_{x_{j}, l_{i}}^{\operatorname{mrc}} t_{l_{i}}} \prod_{l_{i}=1}^{S_{i}} \frac{\mathcal{L}_{\overline{\mathrm{F}}_{H_{i}^{\mathrm{mrc}}}}^{-1}\left(t_{l_{i}}\right)}{\left(1+t_{l_{i}} \beta_{i}\right)^{S_{i}-1}} d t_{l_{i}},
$$

as r.v.s $G_{x_{j}, l_{i}}^{\mathrm{mrc}}$ are i.i.d. across streams. Consequently,

$$
\begin{aligned}
& o_{\mathrm{frt}}^{\mathrm{mrc}}=\sum_{i \in \mathcal{K}} 2 \pi \lambda_{i} \int_{0}^{\infty} x_{i} \mathbb{E}_{\left\{\Phi_{j}\right\}} d x_{i} \int_{0}^{\infty} \ldots \int_{0}^{\infty} \prod_{j \in \mathcal{K}} \prod_{x_{j} \in \Phi_{j} / x_{i}} \mathbb{E}_{G_{x_{j}}} \\
& e^{-\beta_{i} \frac{S_{i}}{P_{i}} x_{i}^{\alpha} \frac{P_{j}}{S_{j}}\left\|x_{j}\right\|^{-\alpha} \sum_{l_{i}=1}^{S_{i}} G_{x_{j}, l_{i}}^{\mathrm{mrc}} t_{l_{i}}} \prod_{l_{i}=1}^{S_{i}} \frac{\mathcal{L}_{\overline{\mathrm{F}}_{H_{i}^{m}}}^{-1}\left(t_{l_{i}}\right)}{\left(1+t_{l_{i}} \beta_{i}\right)^{S_{i}-1}} d t_{l_{i}} \\
& =\sum_{i \in \mathcal{K}} 2 \pi \lambda_{i} \int_{0}^{\infty} x_{i} d x_{i} \int_{0}^{\infty} \ldots \int_{0}^{\infty} \prod_{j \in \mathcal{K}} \mathbb{E}_{\Phi_{j}} \prod_{x_{j} \in \Phi_{j} / x_{i}} \mathbb{E}_{G_{x_{j}}^{\mathrm{mrc}}} \\
& e^{-\beta_{i} \frac{S_{i}}{P_{i}} x_{i}^{\alpha} \frac{P_{j}}{S_{j}}\left\|x_{j}\right\|^{-\alpha} \sum_{l_{i}=1}^{S_{i}} G_{x_{j}, l_{i}}^{\operatorname{mrc}} t_{l_{i}}} \prod_{l_{i}=1}^{S_{i}} \frac{\mathcal{L}_{\overline{\mathrm{F}}_{H_{i}}^{\mathrm{mrc}}}^{-1}\left(t_{l_{i}}\right)}{\left(1+t_{l_{i}} \beta_{i}\right)^{S_{i}-1}} d t_{l_{i}} \\
& =\sum_{i \in \mathcal{K}} 2 \pi \lambda_{i} \int_{0}^{\infty} x_{i} d x_{i} \int_{0}^{\infty} \ldots \int_{0}^{\infty} \prod_{l_{i}=1}^{S_{i}} \frac{\mathcal{L}_{\overline{\mathrm{F}}_{H_{i}}^{\text {mrc }}}^{-1}\left(t_{l_{i}}\right)}{\left(1+t_{l_{i}} \beta_{i}\right)^{S_{i}-1}} d t_{l_{i}} \\
& e^{-x_{i}^{2} \tilde{C}(\alpha)\left(\frac{S_{i} \beta_{i}}{P_{i}}\right)^{\check{\alpha}} \sum_{j=1}^{K} \lambda_{j}\left(\frac{P_{j}}{S_{j}}\right)^{\check{\alpha}} \mathbb{E}_{G_{j}^{\operatorname{mrc}}}\left[\left(\sum_{l_{i}=1}^{S_{i}} G_{j, l_{i}}^{\operatorname{mrc}} t_{l_{i}}\right)^{\check{\alpha}}\right],}
\end{aligned}
$$

where in the last step we have applied following formula [17]:

$$
\mathbb{E}_{\Phi_{j}} \prod_{x_{j} \in \Phi_{j}} \mathbb{E}_{h_{x_{j}}} e^{-s_{j}\left\|x_{j}\right\|^{-\alpha} h_{x_{j}}}=e^{-\tilde{C}(\alpha) s_{j}^{\tilde{\alpha}} \mathbb{E}\left[h^{\check{\alpha}}\right]} .
$$

Consequently,

$$
\begin{gathered}
O_{\mathrm{frt}}^{\operatorname{mrc}}=\sum_{i \in \mathcal{K}} 2 \pi \lambda_{i} \int_{0}^{\infty} \ldots \int_{0}^{\infty} \prod_{l_{i}=1}^{S_{i}} \frac{\mathcal{L}_{\overline{\mathrm{F}}_{H_{i}}^{-1}}^{-1}\left(t_{l_{i}}\right)}{\left(1+t_{l_{i}} \beta_{i}\right)^{S_{i}-1}} d t_{l_{i}} \\
\int_{0}^{\infty} x_{i} e^{-x_{i}^{2} \tilde{C}(\alpha)\left(\frac{S_{i} \beta_{i}}{P_{i}}\right)^{\check{\alpha}} \sum_{j=1}^{K} \lambda_{j}\left(\frac{P_{j}}{S_{j}}\right)^{\check{\alpha}} \mathbb{E}_{G_{j}^{\mathrm{mrc}}}\left[\left(\sum_{l_{i}=1}^{S_{i}} G_{j, l_{i}}^{\mathrm{mrc}} t_{l_{i}}\right)^{\check{\alpha}}\right]} d x_{i} \\
=\sum_{i \in \mathcal{K}} \frac{\pi}{\tilde{C}(\alpha)} \lambda_{i}\left(\frac{P_{i}}{S_{i} \beta_{i}}\right)^{\check{\alpha}} \int_{0}^{\infty} \ldots \int_{0}^{\infty} \prod_{l_{i}=1}^{S_{i}} \frac{\mathcal{L}_{\overline{\mathrm{F}}_{H_{i}^{\mathrm{mrc}}}^{-1}}^{-1}\left(t_{l_{i}}\right)}{\left(1+t_{l_{i}} \beta_{i}\right)^{S_{i}-1}} d t_{l_{i}} \\
\times \frac{1}{\sum_{j \in \mathcal{K}} \lambda_{j}\left(\frac{P_{j}}{S_{j}}\right)^{\check{\alpha}} \mathbb{E}_{G_{j}^{\mathrm{mrc}}}\left[\left(\sum_{l_{i}=1}^{S_{i}} G_{j, l_{i}}^{\mathrm{mrc}} t_{l_{i}}\right)^{\check{\alpha}}\right]}
\end{gathered}
$$

Unfortunately, direct evaluation of (9) seems highly untractable. Instead, we in the following resort to the arithmeticgeometric inequality for deriving an upper-bound, i.e.,

$$
\begin{gathered}
\mathbb{E}_{G_{j}^{\mathrm{mrc}}}\left[\left(\sum_{l_{i}=1}^{S_{i}} G_{j, l_{i}}^{\mathrm{mrc}} t_{l_{i}}\right)^{\check{\alpha}}\right] \geq \mathbb{E}_{G_{j}^{\mathrm{mrc}}}\left[S_{i}^{\check{\alpha}}\left(\prod_{l_{i}=1}^{S_{i}} G_{j, l_{i}}^{\mathrm{mrc}} t_{l_{i}}\right)^{\frac{\check{\alpha}}{S_{i}}}\right] \\
=S_{i}^{\check{\alpha}} \mathbb{E}_{G_{j}^{\mathrm{mrc}}}\left[\prod_{l_{i}=1}^{S_{i}}\left(G_{j, l_{i}}^{\mathrm{mrc}}\right)^{\frac{\check{\alpha}}{S_{i}}}\right]\left(\prod_{l_{i}=1}^{S_{i}} t_{l_{i}}\right)^{\frac{\check{\alpha}}{S_{i}}} .
\end{gathered}
$$

Utilizing this, (9) can be upper-bounded by

$$
o_{\mathrm{frt}}^{\operatorname{mrc}} \leq \sum_{i \in \mathcal{K}} \frac{\frac{\pi}{\bar{C}(\alpha)}\left(\frac{P_{i}}{S_{i} \beta_{i}}\right)^{\check{\alpha}} \frac{\lambda_{i}}{S_{i}^{\tilde{\alpha}}}}{\sum_{j=1}^{K} \lambda_{j}\left(\frac{P_{j}}{S_{j}}\right)^{\check{\alpha}} \mathbb{E}_{G_{j}^{m}} \prod_{l_{i}=1}^{S_{i}}\left(G_{j, l_{i}}^{\mathrm{rmc}}\right)^{\frac{\check{\alpha}}{S_{i}}}}
$$

$$
\begin{aligned}
& \times \int_{0}^{\infty} \cdots \int_{0}^{\infty} \prod_{l_{i}=1}^{S_{i}} \frac{{\frac{\tilde{\alpha}}{l_{i}}}^{-\frac{\check{\alpha}}{S_{i}}} \mathcal{L}_{\overline{\mathrm{F}}_{H_{i}^{\mathrm{mrc}}}}^{-1}\left(t_{l_{i}}\right)}{\left(1+t_{l_{i}} \beta_{i}\right)^{S_{i}-1}} d t_{l_{i}}
\end{aligned}
$$

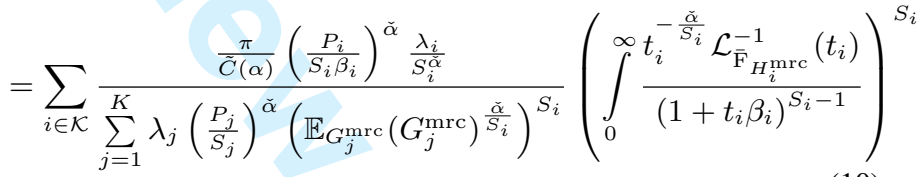

where the last step was due to the fact that r.v.s $G_{x_{j}, l_{i}}^{\mathrm{mrc}}$ are i.i.d. across streams. Note that

$$
\begin{gathered}
\int_{0}^{\infty} \frac{t_{i}^{-\frac{\check{Q}}{S_{i}}} \mathcal{L}_{\overline{\mathrm{F}}_{H_{i}}^{\text {mrc }}}^{-1}\left(t_{i}\right)}{\left(1+t_{i} \beta_{i}\right)^{S_{i}-1}}=\sum_{r_{i}=0}^{N^{r}-1} \frac{1}{r_{i} !} \int_{0}^{\infty} \delta^{\left(r_{i}\right)}\left(t_{i}-1\right) \frac{t_{i}^{-\frac{\check{\alpha}}{S_{i}}}}{\left(1+t_{i} \beta_{i}\right)^{S_{i}-1}} \\
=\left.\sum_{r_{i}=0}^{N^{r}-1} \frac{(-1)^{r_{i}}}{r_{i} !} \frac{d^{r_{i}}}{d t^{r_{i}}} \frac{t^{-\frac{\check{\alpha}}{S_{i}}}}{\left(1+t \beta_{i}\right)^{S_{i}-1}}\right|_{t=1}
\end{gathered}
$$

Also, recalling that r.v.s $G_{x_{j}, l_{i}}^{\mathrm{mrc}}$ are chi-squared with $\operatorname{DoF} 2 S_{j}$, it is straightforward to show that $\mathbb{E}_{G_{j}^{\mathrm{mrc}}}\left(G_{j}^{\mathrm{mrc}}\right)^{\frac{\check{\alpha}}{S_{i}}}=\frac{\Gamma\left(\frac{\check{\alpha}}{S_{i}}+S_{j}\right)}{\Gamma\left(S_{j}\right)}$ By substituting (11) into (10) the desired result is obtained. 